\title{
Engineered Hybrid Scaffolds of Poly(vinyl alcohol)/Bioactive Glass for Potential Bone Engineering Applications: Synthesis, Characterization, Cytocompatibility, and Degradation
}

\author{
Hermes S. Costa, Alexandra A. P. Mansur, Marivalda M. Pereira, and Herman S. Mansur \\ Laboratory of Biomaterials and Tissue Engineering, Department of Metallurgical and Materials Engineering, School of Engineering, \\ Federal University of Minas Gerais, Avenida Antônio Carlos 6627, 31270-901 Belo Horizonte, MG, Brazil \\ Correspondence should be addressed to Herman S. Mansur, hmansur@demet.ufmg.br
}

Received 31 July 2011; Revised 1 September 2011; Accepted 1 September 2011

Academic Editor: Shanfeng Wang

Copyright ( $\odot 2012$ Hermes S. Costa et al. This is an open access article distributed under the Creative Commons Attribution License, which permits unrestricted use, distribution, and reproduction in any medium, provided the original work is properly cited.

\begin{abstract}
The synthesis, characterization, preliminary cytocompatibility, and degradation behavior of the hybrids based on 70\% Poly(vinyl alcohol) and $30 \%$ bioactive glass $\left(58 \mathrm{SiO}_{2}-33 \mathrm{CaO}-9 \mathrm{P}_{2} \mathrm{O}_{5}, \mathrm{BaG}\right)$ with macroporous tridimensional structure is reported for the first time. The effect of glutaraldehyde covalent crosslinker in the organic-inorganic nanostructures produced and, as a consequence, tailoring the hybrids properties was investigated. The PVA/BaG hybrids scaffolds are characterized by Fourier transform infrared spectroscopy (FTIR), scanning electron microscopy (SEM), X-ray diffraction (XRD), and X-ray Microcomputed tomography analysis $(\mu \mathrm{CT})$. Cytotoxicity assessment is performed by the MTT method with VERO cell culture. Additionally, the hybrid in vitro degradation assay is conducted by measuring the mass loss by soaking in deionized water at $37^{\circ} \mathrm{C}$ for up to 21 days. The results have clearly shown that it is possible to modify the PVA/BaG hybrids properties and degradation behavior by engineering the structure using different concentrations of the chemical crosslinker. Moreover, these hybrid crosslinked nanostructures have presented 3D hierarchical pore size architecture varying within $10-450 \mu \mathrm{m}$ and a suitable cytocompatibility for potential use in bone tissue engineering applications.
\end{abstract}

\section{Introduction}

Essentially, the aim of tissue engineering is to restore diseased or damaged tissue to its original state and function, reducing the need for transplants and joint replacements. One of the challenges in tissue engineering is associated with the development of suitable scaffold materials that can act as templates for cell adhesion, growth, and proliferation [13]. More specifically, bone tissue engineering combines cells and biodegradable 3D scaffolds to repair bone tissues. The scaffolds must present an adequate pore size and interconnectivity to promote cell ingrowth, good biocompatibility, and controlled degradation kinetics to match the ratio of replacement by new tissue. Also, the scaffolds should provide an initial biomechanical support until cells generate the extracellular matrix. During the formation, deposition, and organization of that matrix, the scaffold is continuously degraded and metabolized. Thus, the degradation behavior of the produced scaffold is a critically important requirement to be used in tissue engineering applications [3-11]. Nevertheless, most attention has been paid to the synthesis and characterization of the material structure and properties, but comparatively few reports have dealt with the degradation kinetics of the entire system [3-11]. In the last 2-3 decades, a variety of biomaterials have been developed to act as synthetic scaffolds that may guide and stimulate the threedimensional tissue growth [3-14]. Among them, bioactive glasses and related biomaterials have been mostly studied for bone tissue engineering due to their recognized osteoconductive and osteoinductive properties [15-22]. Furthermore, the dissolution products of bioactive glass exert control over genetic factors of bone growth [23]. However, bioactive glasses, compared to cortical and cancellous bones, usually present low mechanical properties, especially in porous 
forms $[10,18]$. This disadvantage significantly restricts the use of these materials in a very broad range of applications. Fortunately, one solution came from mimicking nature, which provides the inspiration to design materials and systems with highly organized structures under dynamically changing conditions. Many of these structures are composed of an intrinsically complex matrix based on organic and inorganic components which produce a natural hybrid material, usually referred to as composites (or nanocomposites). By combining two or more materials in a predesigned manner, a system can be created with properties that are not possible to be attained when considering each of the individual components separately. In addition, the modification of the surface has been used as an alternative route for producing materials with very specific properties for biological applications such as immune diagnosis and protein recognition [24-26]. The development of inorganic-organic composites attempts to create a balance between strength and toughness, in turn improving the characteristics commonly found in the individual components [27]. These composites can be synthesized through a hybridization route, in which two or more components, usually organic and inorganic, are combined [28]. Sol-gel technology allows for the incorporation of polymers of different natures within an inorganic silica bulk, thus producing organic-inorganic hybrid materials [28-31]. Among several choices of polymers, poly(vinyl alcohol) (PVA), a hydrophilic semicrystalline polymer, has been frequently explored as an implant material in wide array of biomedical applications such as drug delivery systems, membranes, wound dressings, artificial skin, and surgical repairs, mainly due to its excellent mechanical strength, biocompatibility, and nontoxicity $[32,33]$. PVA is produced by the polymerization of vinyl acetate to poly(vinyl acetate) (PVAc), and successive hydrolysis to PVA. This reaction, however, is incomplete, resulting in polymers with different degrees of hydrolysis (DH), that is, a copolymer of poly(vinyl alcohol) and poly(vinyl acetate) referred to as poly(vinyl alcohol-co-vinyl acetate). The degree of hydrolysis in PVA affects its physical-chemical properties, such as solubility, hydrophilic/hydrophobic interactions, $\mathrm{pH}$-sensitivity, and viscosity. Additionally, PVA can be crosslinked under acid conditions producing hydrogels with a wide range of swelling and degradation properties for biomedical applications [34].

Recently, hybrid organic-inorganic scaffolds in the poly(vinyl alcohol)/bioactive glass (PVA/BaG) system have been prepared through the sol-gel process [30,35-38], by hydrolysis of tetraethyl orthosilicate in the presence of an acid solution and a subsequent addition of calcium chloride. A solution of PVA, surfactant, and hydrofluoric acid were added to the sol, and the mixture vigorously agitated until the foam had formed. The foam was placed in containers, where gelation occurred, and subsequently dried at low temperatures to avoid polymer thermal degradation. They usually contain unreacted species that may cause some cytotoxicity. This concern was also addressed in previous works in which potentially toxic species remaining within the hybrid structure were blocked by immersion in buffered solutions [37, 38]. Afterward, focusing on applying these materials in tissue engineering, it is crucial to control their degradation behavior based on the designed nanostructure. A versatile method to obtain biomaterials with controlled degradation rates involves the chemical crosslinking of the polymer network [34]. Recent results have demonstrated that an appropriate breakdown of the synthetic biomaterial, as compared to nondegradable hydrogel constructs, can improve healing and increase tissue functions [39]. In addition, the ability to alter physical properties, such as porosity, permeability, and mechanical strength of degradable hydrogels and hybrids, is an integral part of biomaterials design, as they influence cellular growth required for tissue engineering applications [40].

As previously mentioned, our research group has been very active in studying the synthesis and characterization of hybrids based on PVA-modified polymeric networks [25, $30,35-38,41]$ to be used as scaffolds aimed at tissue engineering applications. Nevertheless, to the best of our knowledge, no research has been published which addresses the degradation behavior of hybrids made of polymer (PVA) and bioceramic (bioactive glass, $\mathrm{BaG}$ ) with a rather complex nanostructured network. This approach was used in this work in an attempt to modulate the chemical stability of PVA/BaG hybrids. Thus, the main objective of this study was to develop novel organic-inorganic hybrids with different degrees of chemical crosslinking of their nanostructures and characterize the effect on their properties and preliminary degradation behavior.

\section{Materials and Methods}

2.1. Preparation of Hybrid Samples. The processing route used for synthesizing the inorganic-organic hybrids was based on PVA and sol-gel-derived bioactive glass $(\mathrm{BaG})$ as illustrated in Figure 1. The PVA was selected (Aldrich-Sigma) considering key aspects such as the degree of hydrolysis $(\mathrm{DH}=80 \%)$ and molecular weight $(\mathrm{Mw}=9,000-$ $10,000 \mathrm{~g} / \mathrm{mol}$ ) appropriate for biomedical purposes. Deionized water (DI-water, Millipore Simplicity) with resistivity of $18 \mathrm{M} \Omega \cdot \mathrm{cm}$ was used in all solution preparations. A PVA aqueous solution was prepared at a $28 \mathrm{wt} \%$ concentration by dissolving the PVA powder in a water bath at $80^{\circ} \mathrm{C}$ under constant stirring for $2 \mathrm{~h}$. The $\mathrm{pH}$ of the solution was adjusted to $2.0 \pm 0.1$ by hydrofluoric acid (HF) $2 \mathrm{~N}$ solution.

2.1.1. Preparation of the Starting Bioactive Glass Solution. The synthesis of hybrids based on PVA and inorganic glass has been the subject of investigation of our group in previous reports [30, 36-38]. Here, for clarity sake, just the most relevant parameters will be described. Thus, the starting sol solution with a designed composition of $58 \mathrm{wt} \% \mathrm{SiO}_{2}-33 \mathrm{wt} \%$ CaO-9 wt $\% \mathrm{P}_{2} \mathrm{O}_{5}$ was synthesized by mixing tetraethoxysilane (TEOS), DI water, triethylphosphate (TEP), and calcium chloride together with the hydrofluoric acid $2 \mathrm{~N}$ solution. The $\mathrm{H}_{2} \mathrm{O}$ : TEOS molar ratio used was 12 : 1 .

2.1.2. Preparation of the PVA-Bioactive Glass Hybrid Foams. The prepared hybrid composition consisted of $30 \mathrm{wt} \%$ glass 


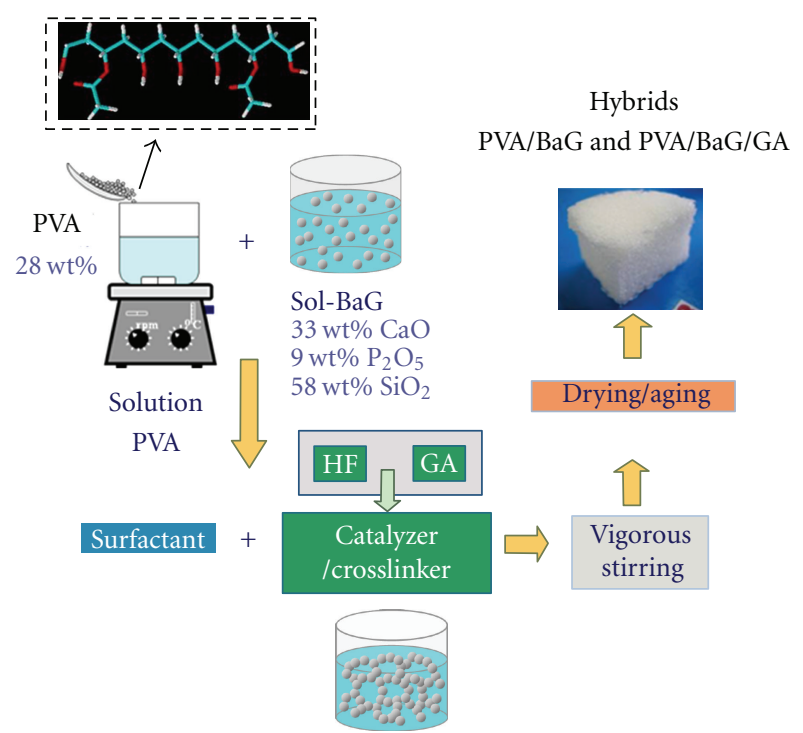

FIGURE 1: Flow chart of the procedure to synthesize PVA/BaG/GA hybrids.

and $70 \mathrm{wt} \%$ polymer. The hybrids were obtained using a procedure similar to that described in previous work from our research group $[36,38]$. In short, an appropriate amount of the starting glass precursors was added to the PVA solution and stirred for five minutes. The surfactant, sodium laureate sulfate (SLS, Oxiteno $27 \% \mathrm{v} / \mathrm{v}$ ) as a foaming agent, and HF $10 \% \mathrm{v} / \mathrm{v}$ solution as a gelling agent were added to the resulting solution. The mixture was foamed by vigorous agitation. The foams were cast shortly before gelation in plastic containers and sealed. The samples were aged at $40^{\circ} \mathrm{C}$ for 3 days and then dried at $40^{\circ} \mathrm{C}$ for 7 days. These hybrids were referred to as $\mathrm{PVA} / \mathrm{BaG}$ samples.

2.1.3. Crosslinking and Stabilization. Glutaraldehyde (GA, Sigma $25 \mathrm{wt} \%$ aqueous solution) was used as a bifunctional chemical crosslinker. Calculated amounts of GA were added, resulting in concentrations of $1.0 \%$ and $5.0 \%$ regarding to the mass of PVA. So, PVA/GA solutions were used to prepare the PVA/BaG/GA samples in a similar procedure as described above for the PVA/BaG foams, as they were casted and dried in the same way. Another set of samples was prepared utilizing a stabilizing step to reduce the acidity of the hybrids that can lead to less cytotoxic biomaterials $[29,30]$. Briefly, samples prepared with $5.0 \%$ of GA (PVA/BaG/GA-5.0\%) were immersed for 10 hours in an $\mathrm{NH}_{4} \mathrm{OH}$ solution $0.3 \mathrm{~mol} \cdot \mathrm{L}^{-1}$. The neutralization process was carried out based on the proportion of $100 \mathrm{~mL}$ of stabilizing solution per $1.0 \mathrm{~g}$ of dried hybrid sample at the temperature of $25 \pm 2{ }^{\circ} \mathrm{C}$. Prior to biological assays, all synthesized samples were washed three times in DI water and dried at room temperature. In the sequence, the hybrids were sterilized with ethylene oxide.

\subsection{Structural and Mechanical Characterization of Hybrids}

2.2.1. Chemical Characterization by FTIR Spectroscopy. FTIR spectra were obtained within the range $600-4000 \mathrm{~cm}^{-1}$
(Perkin-Elmer, Paragon 1000), using the attenuated total reflectance spectroscopy method (ATR-FTIR). Hybrid samples were placed on the ATR crystal prism ( $\mathrm{ZnSe})$, and 64 scans were acquired at $2 \mathrm{~cm}^{-1}$ resolution with the subtraction of background.

2.2.2. Crystallinity and Phase Characterization by X-Ray Diffraction. X-ray diffraction (XRD) patterns were obtained from hybrid PVA/BaG and PVA/BaG/GA using $\mathrm{CuK} \alpha$ radiation with $\lambda=1.54056 \AA$ (PHILIPS, PW1710). XRD analyses were conducted in the $2 \theta$ range from 3.03 to $89.91^{\circ}$ with steps of $0.06^{\circ}$. The major peaks were identified using the "Joint Committee on Powder Diffraction Standards." Samples for XRD analysis were obtained as films, at a thickness of approximately $100 \mu \mathrm{m}$, by pressing $1 \mathrm{~mm}$ thick foams.

2.2.3. Scanning Electron Microscopy (SEM). To evaluate the pore morphology and macropore size distribution, SEM images were taken from organic-inorganic hybrids with a JSM 6360LV (JEOL/NORAN) microscope. Prior to examination, samples were coated with a thin gold film by sputtering. Images of secondary electrons (SE) were obtained using an accelerating voltage of $10-15 \mathrm{kV}$. Pore size distribution analysis was performed using the Quantikov (Version.1.0, Dr. L. C. M. Pinto, CDTN-Brazil) image analysis software.

2.2.4. X-Ray Microcomputed Tomography Analysis ( $\mu C T)$. The structural analysis of cylindrical samples ( $4 \mathrm{~mm}$ diameter) of PVA/BaG/5\% GA was performed on a 3D microtomographer (Phoenix X-Ray Systems and Services GmbH), with a $7 \mathrm{~mm}$ voxel resolution. The 3D image analysis involved the $\mu \mathrm{CT}$ image conversion from the gray-scale images, according to the proper development and use of mathematical operators, which were developed to verify the sample morphology.

2.2.5. Mechanical Properties. Bones are often submitted to compression stress in the body. It has been broadly accepted by the research community to perform compression assays for evaluating biomaterials for potential use as bone repair. For that reason, the mechanical behavior of the composites was evaluated by compression tests. Specimens were evenly cut from the most homogeneous region of the foam to form blocks measuring $10 \times 10 \times 10 \mathrm{~mm}^{3}$. These samples were positioned between parallel plates using equipment EMIC DL 3000 and compressed with a crosshead speed of $0.5 \mathrm{~mm} \cdot \mathrm{min}^{-1}$ and a $2.0 \mathrm{kN}$ load cell. At least five samples $(n=5)$ of each hybrid system were measured and the results averaged. The elastic modulus was calculated as the slope of the initial linear portion of the stress-strain curve. The yield strength was determined from the cross point of the two tangents on the stress-strain curve at the yield point.

2.3. Degradation Behavior-In Vitro Assay. The extent of the degradation process is usually estimated from measurements of mass loss. Thus, the evaluation of the effect of crosslinking on the degradation behavior of the hybrid foams was performed by measuring the mass loss of samples that had 
been chemically modified with different amounts of GA ( $0 \%$, $1.0 \%$, and $5.0 \%$ ) upon immersion in water for different time periods. In addition, hybrids previously neutralized with $\mathrm{NH}_{4} \mathrm{OH}$ solution were also analyzed ( $\left.\mathrm{GA}=5.0 \%\right)$. The samples were prepared as discs with dimensions of $40 \mathrm{~mm}$ diameter by $2 \mathrm{~mm}$ thickness and a diameter/thickness ratio of 20 . The solution volume was chosen to provide a surface of circular section/volume at a solution ratio of $0.1 \mathrm{~cm}^{-1}$. Three samples for each composition $(n=3)$ and time period were cut and weighed six times, the average being considered the equilibrium humid mass. Samples were then kept in a desiccator with silica gel for 24 hours under vacuum, at a temperature of $25 \pm 5^{\circ} \mathrm{C}$. The samples were weighed again, and the procedure was repeated until the equilibrium had been reached. Samples were immersed in DI water and maintained for periods of 2, 7, 14, and 21 days, after which time they were again dried under vacuum and weighed until the equilibrium had been reached. The mass loss was calculated according to

$$
\Delta M=\frac{M_{\mathrm{Si}}-M_{\mathrm{Sf}}}{M_{\mathrm{Si}}} \times 100 \%,
$$

where, $M_{\mathrm{Si}}$ is the dried weight before immersion, and $M_{\mathrm{Sf}}$ is the dried weight after a given immersion time in the degradation medium.

\subsection{Cytotoxicity Assessment_-In Vitro}

2.4.1. MTT Assay. The preliminary cytocompatibility response of the produced hybrids was accessed by the MTT [3-(4,5-dimethylthiazol-2-yl)-2,5-diphenyltetrazolium bromide] assay. The MTT assay was first described by Mosmann in 1983. It is based on the ability of a mitochondrial dehydrogenase enzyme from viable cells to cleave the tetrazolium rings of the pale yellow MTT and form dark blue formazan crystals which is largely impermeable to cell membranes, thus resulting in its accumulation within healthy cells. Solubilization of the cells by the addition of a detergent results in the liberation of the crystals which are solubilized. The number of surviving cells is directly proportional to the level of the formazan product created. The color can then be quantified using a simple colorimetric assay. The results can be read on a multiwell scanning spectrophotometer (ELISA reader) $[42,43]$.

Thus, hybrid scaffolds disks (diameter $=5 \mathrm{~mm}$, thickness $=2 \mathrm{~mm}$ ) were presterilized by means of exposure to saturated steam of ethylene oxide before the MTT assay. The cell viability assay was performed in 5 replicates $(n=$ 5) for each material. The hybrid samples were placed at the bottom of 96-well standard polystyrene microplates (Sarstedt, USA), soaked in $250 \mu \mathrm{L} /$ well minimum essential medium eagle (MEM), and maintained in an incubator at $37^{\circ} \mathrm{C}$ with $5 \% \mathrm{CO}_{2}$ for 24 hours. Next, the resulting solutions were extracted from these wells, and the $\mathrm{pH}$ was measured (buffered medium, $\mathrm{pH}>5.5$ ) before allowing any cell contact. In the sequence, VERO cells (ATCC CCL-81 cell culture isolated from kidney epithelial cells extracted from African green monkeys) at the concentration of $5 \times 10^{5}$ cells $/ \mathrm{mL}$ were seeded to the wells in direct contact with the hybrid scaffolds. Similarly, VERO cells were also placed into the microplate wells used as the references, positive and negative controls of the experiments. Triton X-100 (0.1\%, v/v) was used as negative control (medium known as highly toxic to cells) and the VERO cell culture as the positive control. Then, MEM medium supplemented with $2 \mathrm{mM}$ L-glutamine and $10 \%$ fetal bovine serum (FBS) was added to all the wells, and the system was incubated at $37^{\circ} \mathrm{C}$ with $5 \% \mathrm{CO}_{2}$ for 24 hours. After that, the supernatant from wells was removed and replaced with $200 \mu \mathrm{L} /$ well of fresh MEM/FBS medium and $30 \mu \mathrm{L} /$ well of MTT reagent. Subsequently, the microplate was reincubated for 4 hours with $5 \% \mathrm{CO}_{2}$ at $37^{\circ} \mathrm{C}$ and protected from light. After the incubation period, $35 \mu \mathrm{L} /$ well of sodium dodecyl sulfate (SDS) with $10 \% \mathrm{HCl}$ was added, and the solution was carefully homogenized and reincubated for another $14-16$ hours $\left(5 \% \mathrm{CO}_{2}, 37^{\circ} \mathrm{C}\right.$, protected from light). Finally, the supernatant (clear of sediment or cells) from each well $(\mathrm{V}=100 \mu \mathrm{L} /$ well $)$ was collected and transferred to the respective well of a new blank 96-well microplate where the absorbance was measured at wavelength $\lambda=595 \mathrm{~nm}$. The absorbance background (empty well from the microplate, with no medium or cells) was subtracted from all readings. To test the significance of the observed differences between the groups, a statistical evaluation was carried out using a one-way analysis of variance (ANOVA) with $95 \%$ confidence intervals. The results are expressed as the mean value of 5 replicates \pm standard deviation $(\mathrm{SD})$.

2.4.2. Cell Adhesion-Spreading Assay. Cell viability was evaluated by spreading and attachment assays in order to examine their morphology, adhesion, and spreading behavior. VERO cells were plated at $6 \times 10^{4}$ density on the hosting scaffolds. Cell spreading was evaluated by scanning electron microscopy (SEM, JEOL/Noran, JSM 6360LV) of the specimens after culturing for 2 hours. Before microscopy analysis, samples were fixed with $2 \%$ glutaraldehyde for 16 hours and dehydrated by passing through a series of alcohol solutions (crescent ethanol-water, 20\%; 50\%; 90\%; $100 \% \mathrm{v} / \mathrm{v})$. Then, they were dried in nitrogen flowing reactor for 4 hours and outgassed in vacuum desiccator for 12 hours. Before examination, the samples were sputtered with a thin layer of gold at low deposition rate and placed at the maximum distance from the target to prevent damages. The images were obtained using an accelerating voltage of 10$15 \mathrm{kV}$.

\section{Results and Discussion}

\subsection{Characterization of Hybrids}

3.1.1. Chemical Characterization by FTIR Spectroscopy. Fourier transform infrared spectroscopy (FTIR) was used to characterize the presence of specific chemical groups in the PVA/BaG and PVA/BaG/GA hybrid samples. IR spectra of pure PVA films, bioactive glass $(\mathrm{BaG})$, and the hybrid porous scaffold (PVA/BaG) are summarized in Figure 2(a). These spectra were used as reference for the contribution of each 


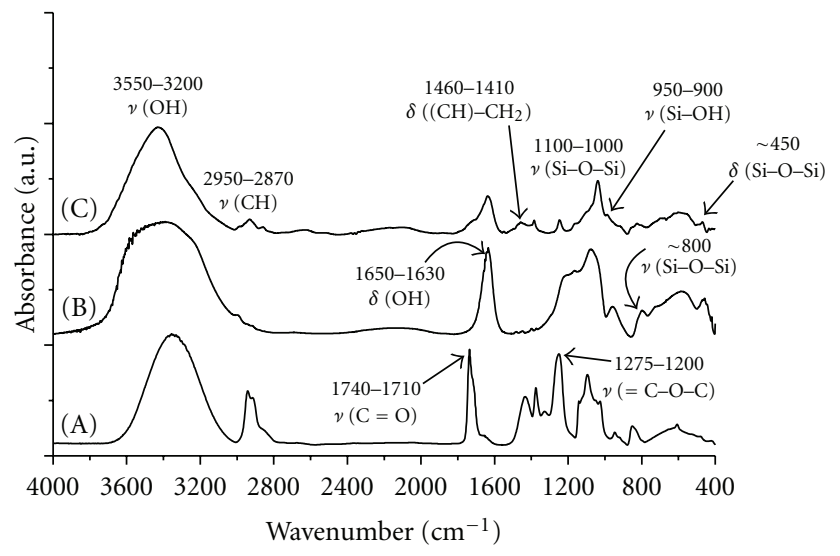

(a)

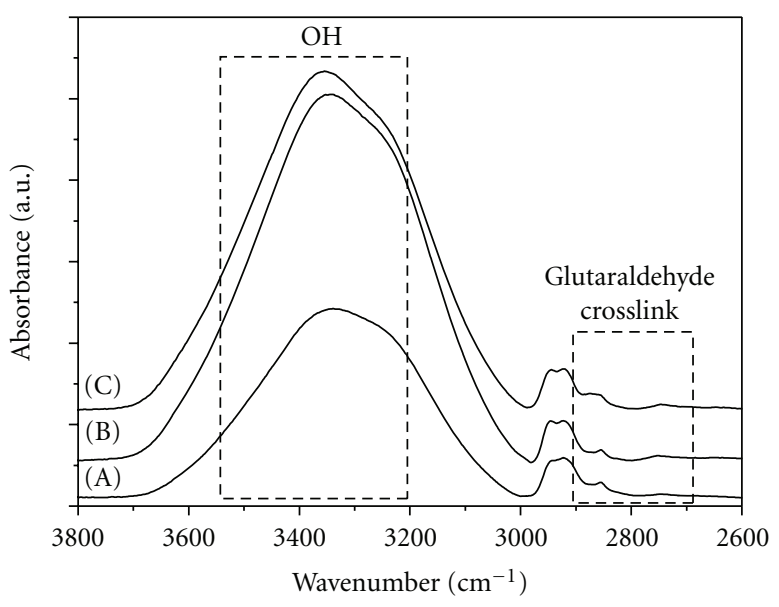

(b)

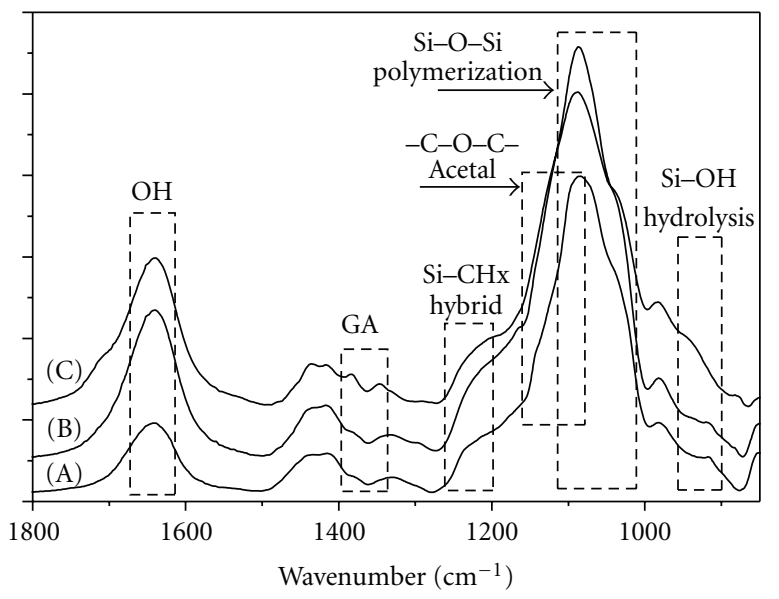

(c)

FIGURE 2: (a) FTIR spectra of (A) PVA films; (B) bioactive glass scaffold; (C) hybrid scaffold of PVA/BaG. (b) FTIR spectra range from 3800 to $2600 \mathrm{~cm}^{-1}$ of PVA/BaG/GA hybrids (A) uncrosslinked (0\%); crosslinked with glutaraldehyde (B) $1.0 \%$ and (C) $5.0 \%$. (c) FTIR spectra range from 1800 to $850 \mathrm{~cm}^{-1}$ of PVA/BaG/GA hybrids (A) uncrosslinked (0\%); crosslinked with glutaraldehyde (B) $1.0 \%$ and (C) $5.0 \%$.

and every component on the final produced hybrid network. Hence, the broad band observed from 3200 to $3550 \mathrm{~cm}^{-1}$ in the PVA spectra (Figure 2(a)-(A)) may be assigned to hydroxyls $(\nu \mathrm{OH})$ stretching due to the strong hydrogen bond of intramolecular and intermolecular type [25, 30, 41, 44]. Also, the strong band at $2870-2950 \mathrm{~cm}^{-1}$ was attributed to alkyl stretching mode $(\nu \mathrm{CH})$. The absorption bands ranging from 1710 to $1750 \mathrm{~cm}^{-1}$ and 1200 to $1275 \mathrm{~cm}^{-1}$ arise due to the stretching vibration of carbonyl $(\nu \mathrm{C}=\mathrm{O})$ and ester, respectively, from the vinyl acetate group found in partially hydrolyzed PVA polymer ( $\mathrm{DH}=80 \%)$. Some other peaks which can be found related to PVA are located at $1410-1460 \mathrm{~cm}^{-1}$ assigned to $\delta(\mathrm{CH}) \mathrm{CH}_{2} ; 1200-1270 \mathrm{~cm}^{-1}$ of group $v(\mathrm{C}-\mathrm{O})-\mathrm{C}-\mathrm{OH} ; 820-850 \mathrm{~cm}^{-1}$ from alkyl chain backbone $[25,30,41,44]$. In an analogous analysis, the FTIR spectrum of the bioactive glass (BaG, Figure $2(\mathrm{a})-(\mathrm{B})$ ) presented the bands related to $\mathrm{Si}-\mathrm{O}-\mathrm{Si}$ asymmetric and symmetric stretching modes at approximately $1100 \mathrm{~cm}^{-1}$ and $800 \mathrm{~cm}^{-1}$, respectively [45-48]. A characteristic absorption band observed in silica gel is located at $1640 \mathrm{~cm}^{-1}$ and is attributed to the deformation mode of adsorbed molecular water in the pores $[45,46]$. Also, the vibrational band at 900-950 $\mathrm{cm}^{-1}$ has been credited to the presence of silanol groups $(\mathrm{Si}-\mathrm{OH})$ usually found in silica synthesized via solgel method. The overall contribution of major groups from each component on the hybrid network can be identified in Figure 2(a)-(C). The FTIR spectrum has presented broad bands in the frequency ranging from 3100 to $3650 \mathrm{~cm}^{-1}$ attributed to both contributions of hydroxyls (PVA) and silanols $(\mathrm{BaG})$. There is an overlapping of the bands in the range from 900 to $1500 \mathrm{~cm}^{-1}$ derived from the bioactive glass and the PVA components $[25,30,41,44]$. It is worth noting that the hybrid formation leads to the broadening of the bands related to vinyl acetate copolymer, that almost disappear as a consequence of the hydrogen bonds involving $\mathrm{C}=\mathrm{O}$ groups and silanol groups in silicate networks [49].

The FTIR spectra of PVA/BaG hybrids before (curve (a)) and after chemical crosslinking with glutaraldehyde $(1.0 \%$, curve (b); 5.0\%, curve (c)) are shown in Figures 2(b) and 2(c). The effect of the GA crosslinker was highlighted in 
different regions of the spectra presented in Figure 2(b). The hydroxyls reaction with aldehydes forming hemiacetals and acetals are evidenced at the range from 3200 to $3550 \mathrm{~cm}^{-1}$ $(-\mathrm{OH})$ and from 2750 to $2850 \mathrm{~cm}^{-1}$ (GA duplet). In the same way, Figure 2(c) shows the FTIR spectra region mostly related to the inorganic species of the hybrid network affected by the crosslinking, for instance, the $\mathrm{Si}-\mathrm{O}-\mathrm{Si}$ bands from solgel process and the very relevant hybrid group $\mathrm{Si}-\mathrm{CH}_{x}(1200-$ $\left.1260 \mathrm{~cm}^{-1}\right)$. However, a broader absorption band (1085$\left.1150 \mathrm{~cm}^{-1}\right)$ that is attributed to the ether $(\mathrm{C}-\mathrm{O})$ and acetal and hemiacetal bridges $(-\mathrm{C}-\mathrm{O}-\mathrm{C}-$ ) bands by the crosslinking reaction of PVA with GA can be observed [25]. As mentioned, it was feasible to observe by FTIR spectroscopy the relative contribution of the organic (PVA), inorganic ( $\mathrm{Si}-$ $\mathrm{O}-\mathrm{Si}$ ) components and the chemical crosslinker (GA) in the different hybrids produced.

It is well known that FTIR is certainly a qualitative spectroscopic method for investigating chemical functionalities in materials. Nevertheless, with some fundaments and proper assumptions, it may be a powerful tool to conduct semiquantitative analysis by considering the ratio of intensities (height or peak areas). The FTIR spectra were normalized using $\mathrm{CH}_{2}$ bending mode at $1450 \mathrm{~cm}^{-1}$ before performing the calculations with the intensities. This band associated with the $\mathrm{CH}_{2}$ bending mode is relatively unchanged by adjacent chemical alterations [24, 25, 30, 34]. Consequently, the effect of crosslinking on the nanostructure of the hybrids was assessed by analyzing the changes on the relative intensities of relevant specific FTIR bands. This means that major bands associated with the polymer, the bioactive glass, and the network modifier were compared for different contents of crosslinking agent added.

In fact, these relative intensities of FTIR bands have offered the possibility of evaluating the actual degree of crosslinking and, as a result, the density of covalent bonds formed in the chemically modified hybrid network as presented in Figure 3. Thus, it can be observed in Figure 3(b) that the relative intensities $\left(\mathrm{I}_{\mathrm{C}-\mathrm{O}-\mathrm{C}}\right)$ associated with the formation of acetal and hemiacetals $\left(1085-1150 \mathrm{~cm}^{-1}\right)$ increased in the PVA/BaG hybrids crosslinked with glutaraldehyde (1.0 and 5.0\%) compared to noncrosslinked ones. As expected, the covalent chemical crosslinking has taken place by the reaction of bifunctional aldehyde (GA) with the hydroxyls groups of PVA. Also, that effect was not evident by increasing the GA concentration from $1.0 \%$ to $5.0 \%$, perhaps limited by some saturation of reacting sites available and the restriction in the mobility of PVA chains caused by the network crosslinking.

For the inorganic phase, the bands chosen were 10001100 and $900-950 \mathrm{~cm}^{-1}$, associated with the bridging Si$\mathrm{O}-\mathrm{Si}\left(\mathrm{I}_{\mathrm{Si}-\mathrm{O}-\mathrm{Si}}\right)$ and $\mathrm{Si}-\mathrm{OH}\left(\mathrm{I}_{\mathrm{SiOH}}\right)$ bonds, respectively. The intensity ratios for bands $\mathrm{I}_{\mathrm{Si}-\mathrm{O}-\mathrm{Si}}$ and $\mathrm{I}_{\mathrm{SiOH}}$ as a function of the concentration of crosslinking agent added are presented in Figures 3(c) and 3(a), respectively. It can be observed that the peak intensity ratio increased due to the formation of $\mathrm{Si}-\mathrm{O}-\mathrm{Si}$ bonds $\left(\mathrm{I}_{\mathrm{Si}-\mathrm{O}-\mathrm{Si}}\right)$ for $1.0 \%$ GA compared to the noncrosslinked hybrids. It is suggested that the crosslinking reaction of PVA reduced the amount of hydroxyls (acetal formation $=$ alcohol + aldehyde $)$, reducing the sites available

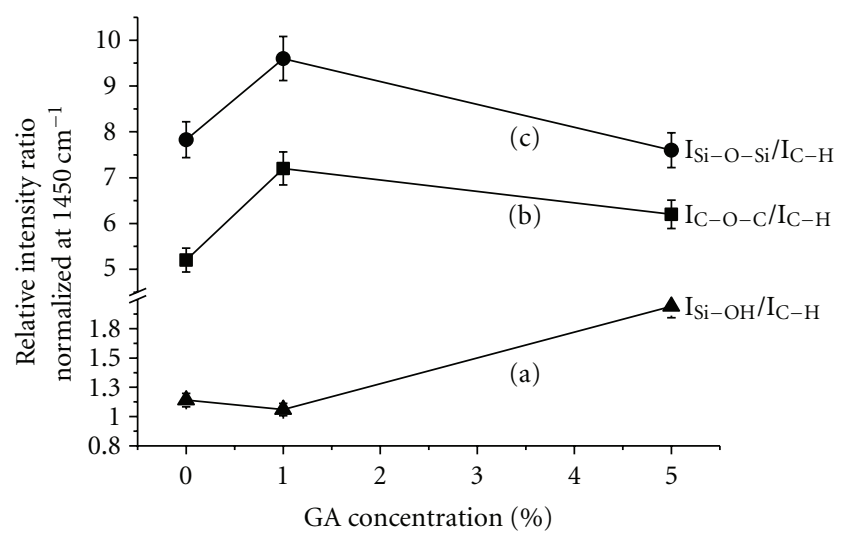

FIGURE 3: Hybrid nanostructure modification by chemical crosslinking (GA). Relative intensity ratios for specific peaks of the FTIR spectra as a function glutaraldehyde concentration in hybrids PVA/BaG/GA: (a) $\mathrm{I}_{\mathrm{Si}-\mathrm{OH}} / \mathrm{I}_{\mathrm{C}-\mathrm{H}}$; (b) $\mathrm{I}_{\mathrm{C}-\mathrm{O}-\mathrm{C}} / \mathrm{I}_{\mathrm{C}-\mathrm{H}}$; (c) $\mathrm{I}_{\mathrm{Si}-\mathrm{O}-\mathrm{Si}} / \mathrm{I}_{\mathrm{C}-\mathrm{H}}$.

for hydrogen bonds formation between PVA and silanol groups. Therefore, that would have favored an increase in the inorganic polycondensation reactions leading to the formation of silicate network $(\mathrm{Si}-\mathrm{OH}+\mathrm{Si}-\mathrm{OH}=\mathrm{Si}-\mathrm{O}-$ $\mathrm{Si}$ ) (Figure 3(c)). On the contrary, the peak intensity ratio related to the silanols (nonbridging oxygen, $\mathrm{I}_{\mathrm{SiOH}}$ ) remained practically constant for noncrosslinked and $1.0 \%$ crosslinked hybrids but drastically increased for hybrids with 5.0\% GA (Figure 3(a)). This trend can be attributed to the fact that at very high GA concentration (5.0\%), the crosslinking reaction has occurred very intensively, as indicated by a much lower gelation time during the synthesis (less than half the gelation time observed for hybrids produced with no addition of GA) $[36,38]$. As a consequence, the fast gelation of the mixture may have affected the inorganic polycondensation reactions ("rigid frozen structure"), accounted for the reduction of $\mathrm{I}_{\mathrm{Si}-\mathrm{O}-\mathrm{Si}}$ band (Figure 3(c)) for 5.0\% GA, leading to a higher amount of $\mathrm{Si}-\mathrm{OH}$ groups remaining within the hybrid structure and, therefore, to an increase in the value of $\mathrm{I}_{\mathrm{SiOH}}$ (nonbridging in the inorganic network). Moreover, it could be assumed that at very high aldehyde concentration, the stage of the sol-gel process associated with polycondensation was significantly limited by the acetal bridges formed with PVA (alcohol hydroxyls) causing the "excess" of remaining unreacted silanol groups within the $3 \mathrm{D}$ hybrid network.

\subsubsection{Crystallinity and $3 D$ Morphology of $P V A / B a G / G A$} Hybrids. The X-ray diffraction patterns for samples of PVA/BaG crosslinked with GA are presented in Figure 4. It can be observed that the noncrosslinked hybrid has shown mostly an amorphous structure most likely caused by the synergetic effect of bioactive glass and semicrystalline PVA. On the other hand, the chemically crosslinked network indicated a partially crystalline structure. In addition, a crystalline phase of calcium silicate was identified as $\mathrm{CaSiO}_{3}$ (Figure 4(a), bottom) for the hybrid systems covalently crosslinked with the bifunctional modifier (GA). This finding can be attributed to the kinetics of the hybrid structure formation as the crosslinker has preferably reacted with 


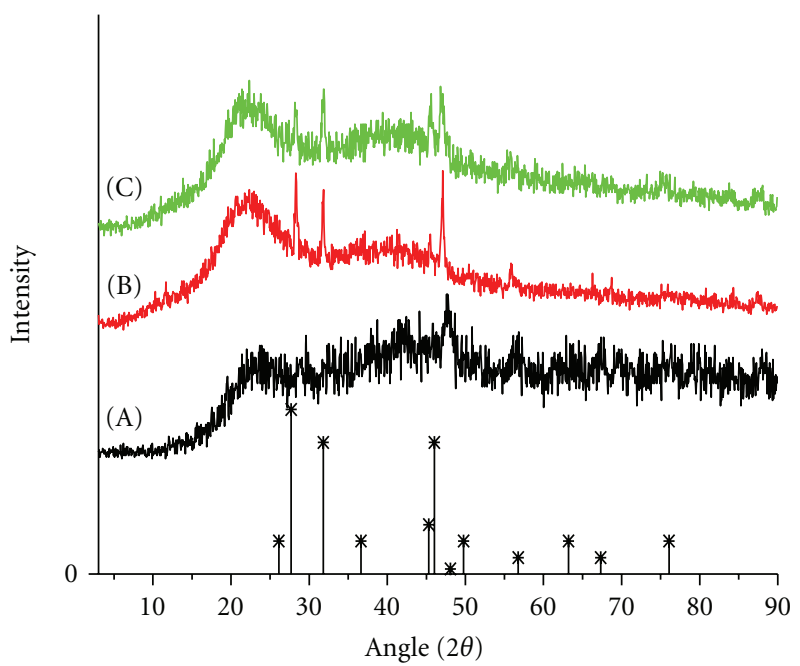

(a)

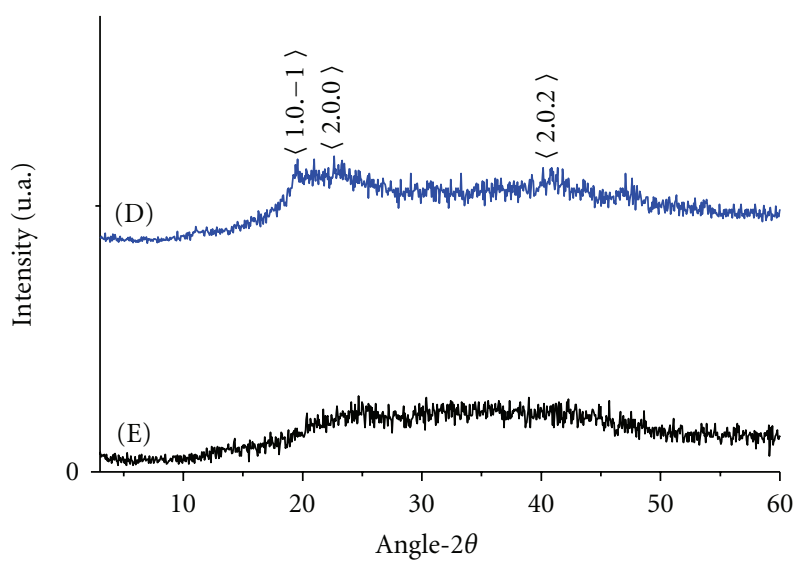

(b)

Figure 4: XRD patterns of hybrids. (a): (A) BaG/PVA, (B) BaG/PVA/1.0\% GA, and (C) BaG/PVA/5.0\% GA, (*) CaSiO 3 crystalline phase. (b): (D) PVA, and (E) bioactive glass (BaG).

TABle 1: Pore size analyses of PVA/BaG/GA hybrids.

\begin{tabular}{|c|c|c|c|c|}
\hline & \multicolumn{4}{|c|}{ Concentration glutaraldehyde (GA, \%) } \\
\hline & 0.0 & 1.0 & 5.0 & $5.0^{*}$ \\
\hline Medium pore size $(\mu \mathrm{m})$ & 450 & 490 & 150 & \\
\hline Maximum pore size $(\mu \mathrm{m})$ & 1100 & 1000 & 450 & 450 \\
\hline Minimum pore size $(\mu \mathrm{m})$ & 220 & 180 & 50 & 100 \\
\hline Interconnections medium size $(\mu \mathrm{m})$ & 75 & 145 & 35 & \\
\hline Interconnections maximum size $(\mu \mathrm{m})$ & 285 & 475 & 100 & 200 \\
\hline Interconnections minimum size $(\mu \mathrm{m})$ & 25 & 35 & 10 & 60 \\
\hline
\end{tabular}

*Values obtained by micro-CT.

alcohol groups from PVA, in turn leading to relatively richer calcium $\left(\mathrm{Ca}^{2+}\right)$ nanodomains, allowing for reactions with silanols $(\mathrm{Si}-\mathrm{OH})$, forming the calcium silicate phase. It should be noted that, preliminarily, this phase is not detrimental to either mechanical or biological properties.

Moving a step further, the 3D structure of the hybrid was characterized by SEM and $\mu \mathrm{CT}$ analyses. The SEM micrographs have shown the macroporous network produced for different amounts of GA added to the PVA/BaG systems (Figures 5(a), 5(b), and 5(c)). Noncrosslinked or crosslinked hybrids with $1.0 \%$ GA have shown a comparable pore structure, in which larger pores (macropore) are interconnected by smaller channels (interconnections). On the other hand, the size range of both macropores and interconnections decreased significantly when the material was crosslinked with a larger amount of GA (5.0\%). The results of the quantitative assessment of the macropore and interconnection size distribution, performed using the image analysis software (Quantikov), are presented in Table 1.
The pore size range and interconnection size range are much lower for the samples crosslinked with 5.0\% GA, as is qualitatively illustrated on the images. The macropore average diameter was $450 \mu \mathrm{m}$ for the PVA/BaG sample (noncrosslinked) and $490 \mu \mathrm{m}$ for the sample crosslinked with $1.0 \%$ GA (PVA/BaG/GA). The average pore diameter fell to $150 \mu \mathrm{m}$ in samples crosslinked with $5.0 \%$ GA. In summary, it is reasonable to affirm that these results have evinced a pronounced effect of the network reticulation by means of covalent chemical modifiers on pore size distribution.

The pore size analysis for the sample crosslinked with 5.0\% GA was also conducted using X-ray microcomputed tomography $(\mu \mathrm{CT})$, a tool that has become more progressively used to obtain three-dimensional (3D) images of scaffold biomaterials, as it allows for a better evaluation of the $3 \mathrm{D}$ pore structure [50]. The $3 \mathrm{D}$ image obtained by $\mu \mathrm{CT}$ is shown in Figure 5(d), and the corresponding pore size distribution and interconnection distribution are presented in Figures 5(e) and 5(f). The $\mu \mathrm{CT}$ image clearly shows 


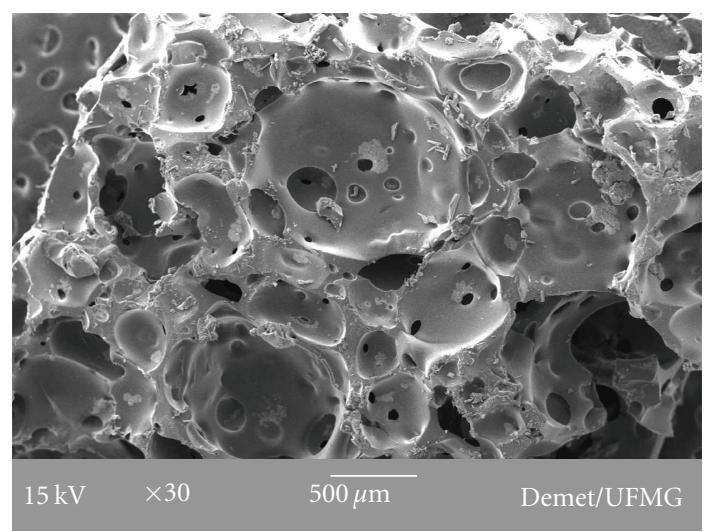

(a)

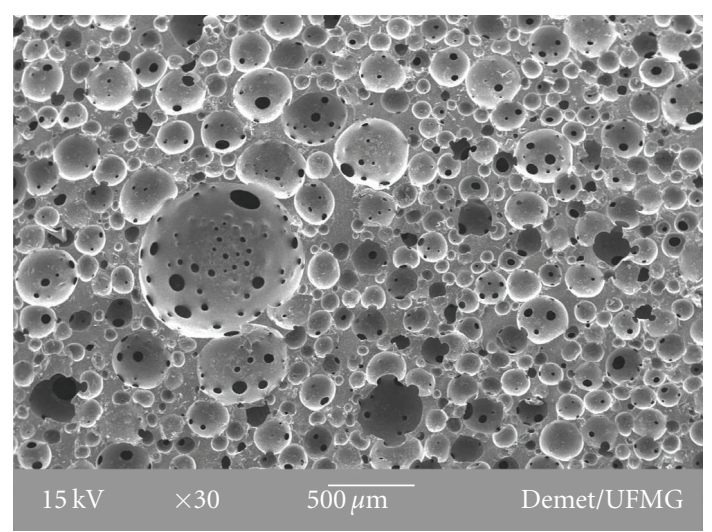

(c)

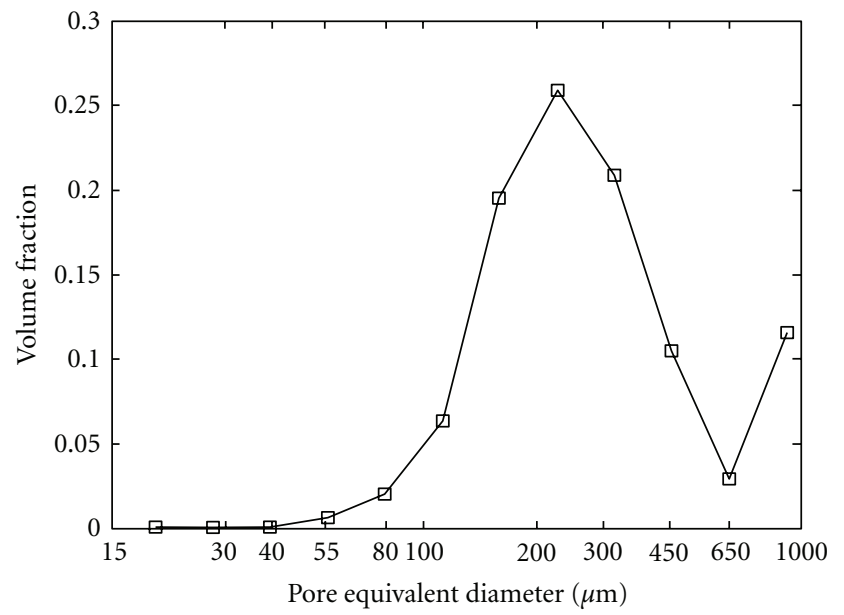

(e)

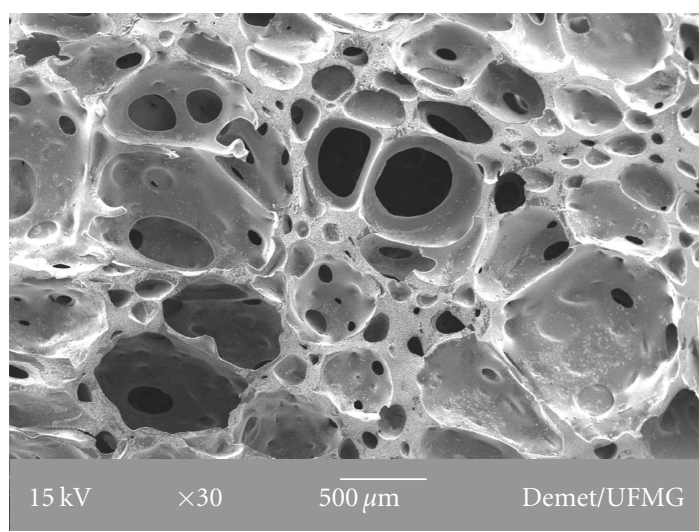

(b)

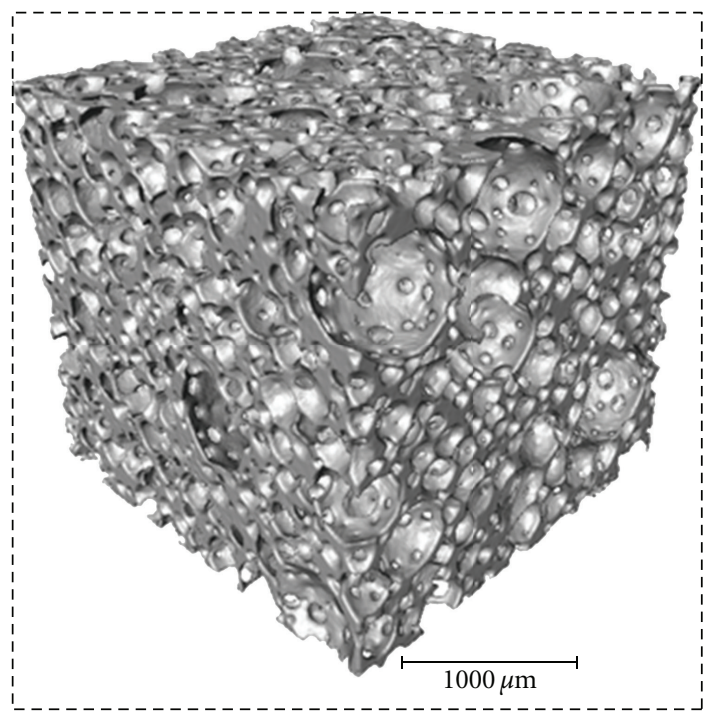

(d)

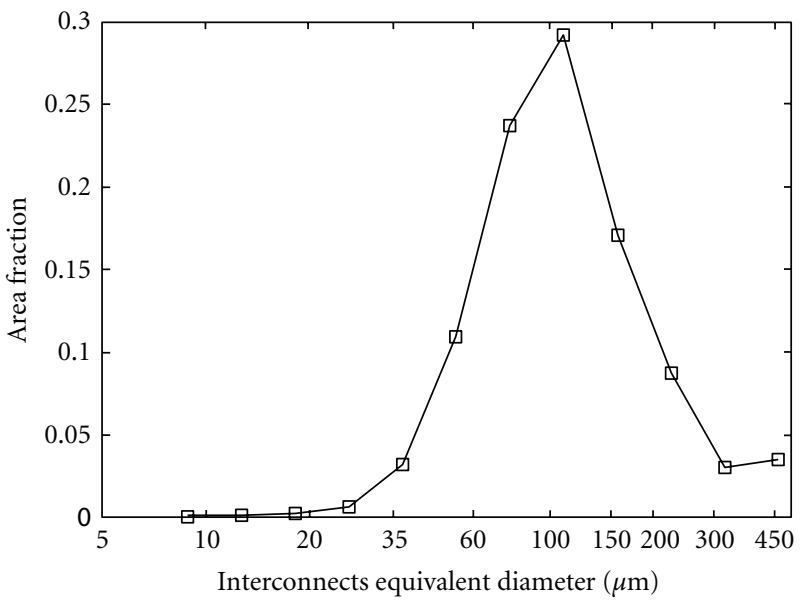

(f)

Figure 5: SEM of PVA/BaG/GA hybrids crosslinked with (a) $0 \%$; (b) $1.0 \%$; (c) $5.0 \%$ of GA. (d) X-Ray microcomputed tomography of sample crosslinked with $5.0 \%$ of glutaraldehyde. Pore size distribution (e) and interconnection size distribution (f) obtained by $\mu \mathrm{CT}$ analysis of the hybrid PVA/BaG crosslinked with $5.0 \%$ glutaraldehyde. 
TABLE 2: Compression yield strength, deformation at yield stress, and elastic compression modulus for PVA/BaG/GA hybrids.

\begin{tabular}{lccc}
\hline$[\mathrm{GA}]$ & $\begin{array}{c}\text { Yield strength } \\
(\mathrm{MPa})\end{array}$ & $\begin{array}{c}\text { Deformation at yield stress } \\
(\%)\end{array}$ & $\begin{array}{c}\text { Elastic compression modulus } \\
(\mathrm{MPa})\end{array}$ \\
\hline $0 \%$ & $2.3 \pm 0.9$ & $7.5 \pm 0.3$ & $0.6 \pm 0.2$ \\
$1.0 \%$ & $6.7 \pm 3.3$ & $9.1 \pm 2.9$ & $0.8 \pm 0.1$ \\
$5.0 \%$ & $9.1 \pm 0.3$ & $14.6 \pm 1.1$ & $0.8 \pm 0.1$ \\
$5.0 \%-\mathrm{NH}_{4} \mathrm{OH}$ & $7.5 \pm 1.1$ & $9.9 \pm 0.9$ & $1.0 \pm 0.1$ \\
Maximum stress for & & $2-12 \mathrm{MPa}$ & \\
trabecular bone $[30,36]$ & & & \\
\hline
\end{tabular}

that the PVA/BaG/5.0\% GA hybrid presents a 3D porous structure with interconnected pores. The distributions show that $80 \%$ of the macropore range from 100 to $450 \mu \mathrm{m}$ with a modal pore size of $227 \mu \mathrm{m}$ (Table 1). The interconnections also show a large distribution, with $80 \%$ ranging from 60 to $200 \mu \mathrm{m}$ and a modal size of $110 \mu \mathrm{m}$. Comparing the results obtained by SEM and $\mu \mathrm{CT}$ for the PVA/BaG/5.0\% GA hybrid, it can be observed that the maximum values for macropore size are similar. However, when comparing the minimum pore size, values measured by SEM and $\mu \mathrm{CT}$ are quite different. This difference may well be related to the difficulty in clearly defining, through SEM images, the difference between macropores and interconnections. As mentioned in the previous section, the reduction in pore and interconnection size for larger concentrations of the crosslinking agent may be explained by assuming that the capacity of the aldehyde groups of GA reacts with the hydroxyl groups present in PVA, thus forming bridges between the polymeric chains and contributing to the faster gelation of the solution. Upon increasing the crosslinking rate, the gelation process is then completed, in turn freezing the structure of the foam before bubbles from vigorous stirring begin to coalesce. In fact, in Figure 5 (A, B, C), an expressive amount of pores with diameters of approximately $50 \mu \mathrm{m}$ in the sample crosslinked with $5.0 \%$ GA can be observed. This result shows that the gelation step plays a crucial role in the dynamics of pore formation. When the gelation time is increased, bubbles introduced before gelation can migrate into the foam and form groups that coalesce to originate large pores before viscosity increases at the gel point, thus stabilizing the material.

3.1.3. Mechanical Characterization. The mechanical response under compression of hybrid samples before (PVA/ $\mathrm{BaG}$ ) and after having been chemically modified by crosslinking (PVA/BaG/GA) is presented in Figure 6. In a general approach, it can be said that the mechanical behavior found for the PVA/BaG hybrids is in accordance with the reported literature for cellular (porous) materials [51]. Typically, the stress-strain curve for a cellular solid in compression is characterized by three regimes (Figure 6(a), regions I, II and III): a linear elastic regime, corresponding to cell edge bending or face stretching; a stress plateau, corresponding to progressive cell collapse by elastic buckling, plastic yielding, or brittle crushing, depending on the nature of the solid from which the material is made; densification, corresponding to the collapse of the cells throughout the material and subsequent loading of the cell edges and faces against one another. In the produced hybrids, a marked change could be observed, particularly in the plastic regime, as the amounts of crosslinking agent increased, which can be attributed to different mechanisms leading to cell collapse. For hybrids with no crosslinking agent added or with only $1.0 \%$ GA added, the cell collapse is due to brittle crushing, although some plastic deformation might occur. The stress plateau became more defined for samples with higher added amounts of GA (5.0\%), which is typical of a more plastic behavior where progressive cell collapse due to plastic yielding can be observed. For these samples, in the final stage, the compression stress increased rapidly with strain, leading to a densification of the foam due to the collapse of the cells. It should be emphasized that all samples were carefully dried in a vacuum before testing, since the high polymer content of the samples determines its hydrophilic character, and that adsorbed water acts as a plasticizer for PVA, influencing the stress-strain behavior. The mechanical properties of all hybrids are summarized in Table 2. An increase in the yield strength (Figure 6(b)), as well as in the deformation at the yield stress (Figure 6(c)), could be observed in direct proportion to the increase in the amount of crosslinking agent added. Hybrids prepared with no crosslinking agents presented a yield strength of approximately $2 \mathrm{MPa}$, close to the lower level of the range measured for the trabecular bone [30]. The addition of 1.0\% GA led to a threefold $(\sim 200 \%)$ increase in the yield strength. The samples prepared with $5.0 \%$ GA presented a yield strength of $9 \mathrm{MPa}$, which is four times higher $(\sim 300 \%)$ than the value obtained for noncrosslinked hybrids. These findings are relevant as far as bone tissue engineering is concerned, where a significant improvement in mechanical properties was achieved, producing only minor changes in the overall $3 \mathrm{D}$ structure. At this stage, it should be emphasized that even at the highest crosslinker concentration (5.0\% GA), the pore size distribution and the connectivity would make it possible to promote cell adhesion, migration, and growth mimicking the trabecular (cancellous) bone structure with comparable mechanical properties. These results are a consequence of the homogeneous $3 \mathrm{D}$ pore structure of the hybrids prepared with $5.0 \%$ GA which are endorsed by the low values for the standard deviation (3\% at yield strength) and SEM image analysis. Nevertheless, it should also be highlighted that the most important effect of the addition of the higher content of crosslinking agents was to change the behavior of 


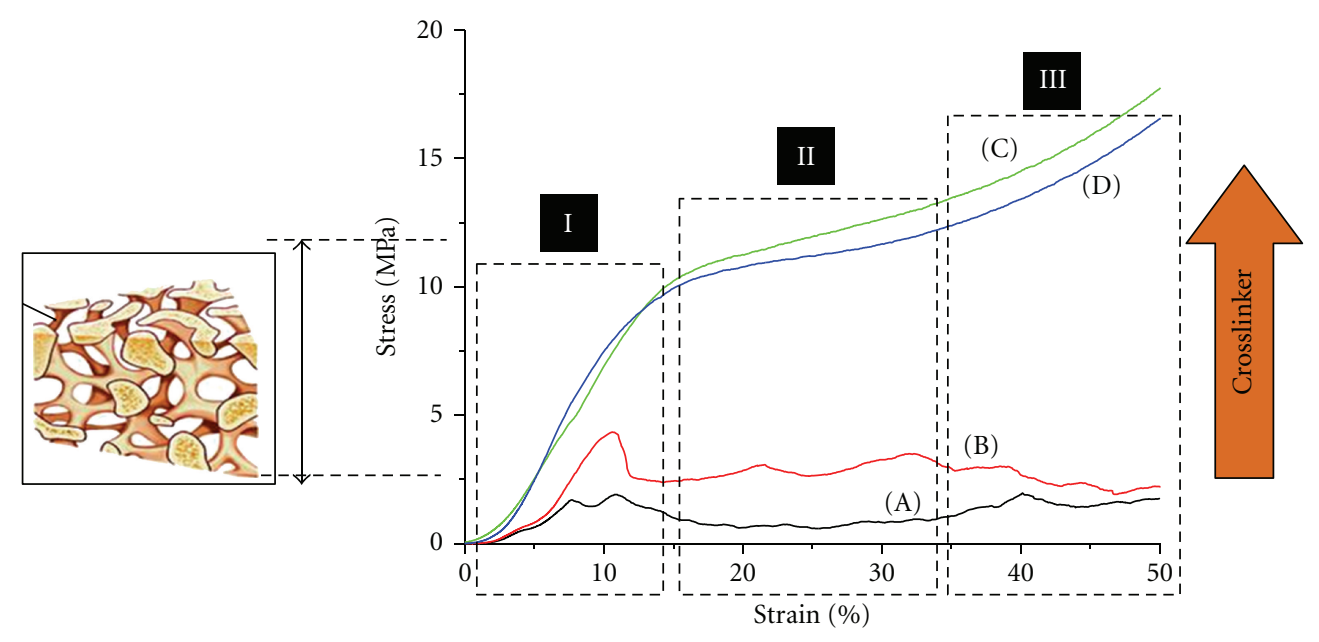

(a)

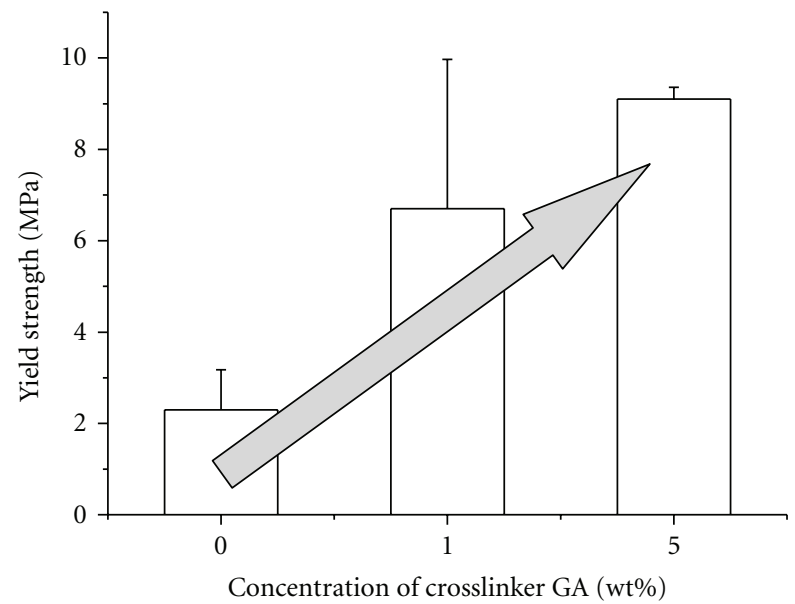

(b)

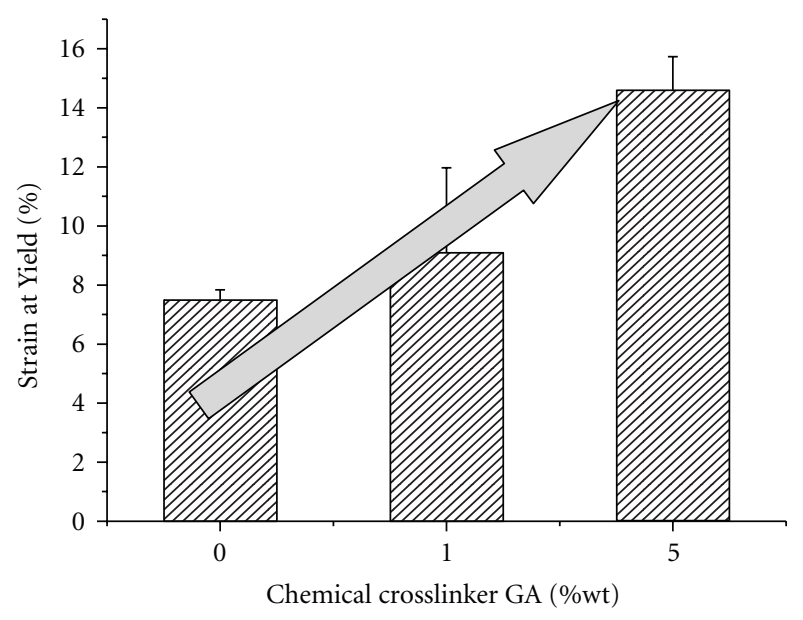

(c)

Figure 6: (a) Stress-strain compression curves obtained for PVA/BaG/GA hybrids crosslinked with (A) 0\%, (B) 1.0\%; (C) 5.0\% of glutaraldehyde, and (D) crosslinked with 5.0\% GA and treated with $\mathrm{NH}_{4} \mathrm{OH}$. Regions I, II and III related to the mechanical behavior of the nanostructured system. (b) Histogram of the yield strength results for PVA/BaG/GA hybrids crosslinked with (A) $0 \%$; (B) $1.0 \%$, (C) $5.0 \%$ glutaraldehyde. (c) Histogram of the strain at yield results for PVA/BaG/GA hybrids crosslinked with (a) $0 \%$; (b) $1.0 \%$, (c) $5.0 \%$ glutaraldehyde.

the porous hybrid to a more plastic deformation. The effect of crosslinking was similar to that observed when increasing the amount of PVA in the hybrid and under varying drying conditions, which determines the residual content of water in the material [52]. In both cases, the mechanism of cell collapse turned to plastic yielding, as the PVA and water acted as the plasticizer of the material. The mechanical characterization of the trabecular bone is very difficult.

3.2. Degradation Behavior: In Vitro Assay. At this point, it is important to present some considerations regarding to the degradation process. Despite being intensively researched, the degradation behavior of polymeric systems is a difficult theme as it may vary a lot from one system to another. One can simply classify them as degradable and "nondegradable" (inert). Nevertheless, the distinction between "degradable" and "non-degradable" polymers is unclear and quite arbitrary, given that all materials, including polymers, will degrade within an appropriate time scale. It is the relation between the time scale of degradation and the timescale of application that appears to make the difference between "degradable" and "non-degradable" polymers. The attribute "degradable" is commonly assigned to materials which degrade during their application or immediately after it. "Non-degradable" polymers are those that require a substantially longer time to degrade than the duration of their application. In addition, the degradation behavior of these systems is quite different, as they are commonly utilized as biomaterials in living organisms. A number of new aspects and properties must be fulfilled by the designed system in order to reach a successful performance under any clinical application. Complexity is taken to the extreme, bearing in mind the degradation process of three-dimensional porous hybrid scaffolds submitted to this dynamic physiological 


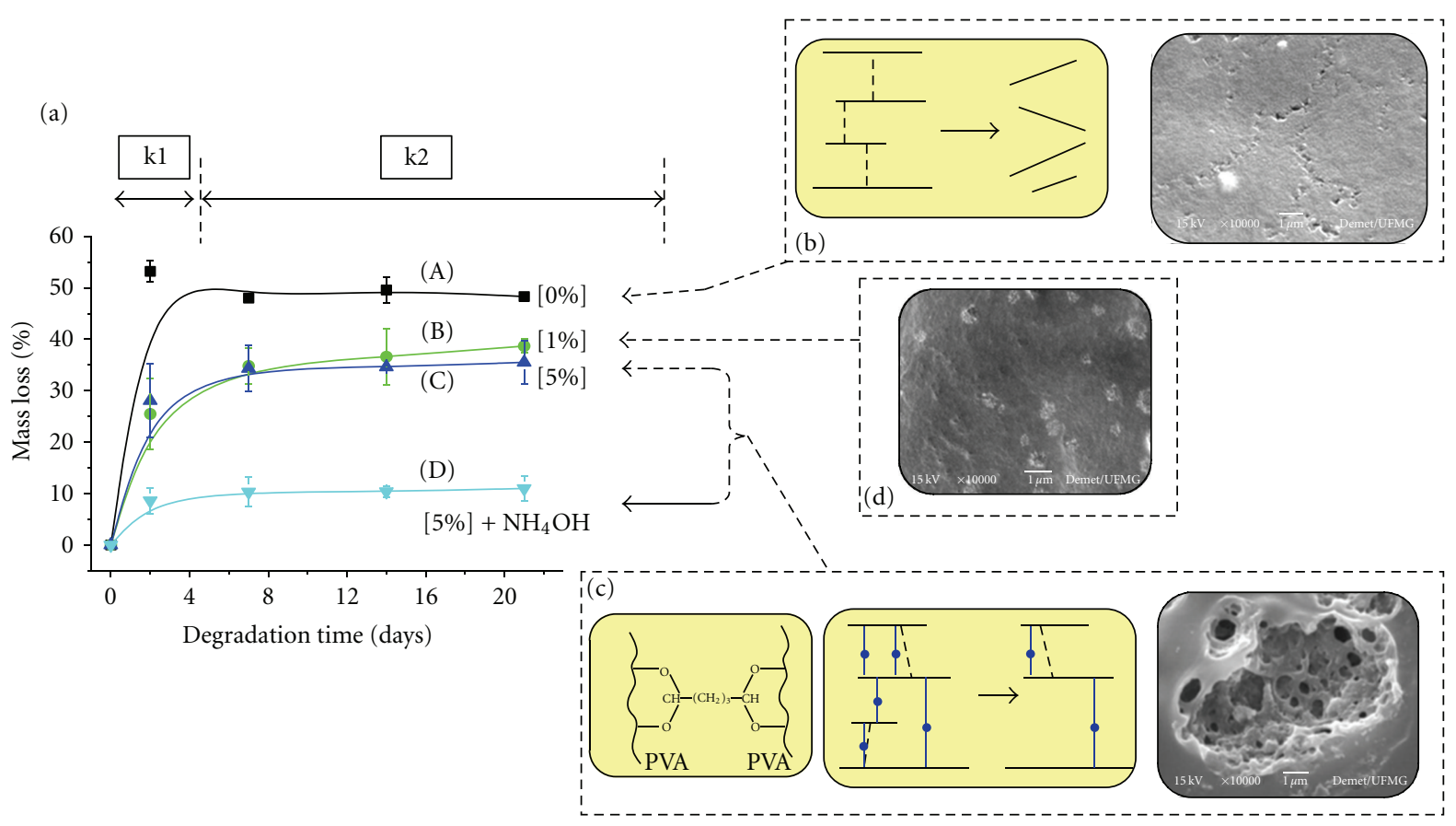

FIGURE 7: (a) Degradation assay in vitro for PVA/BaG/GA hybrids (A) noncrosslinked (0\% GA); crosslinked with (B) $1.0 \%$ and (C) $5.0 \%$ GA; (D) crosslinked with 5.0\% GA and treated with $\mathrm{NH}_{4} \mathrm{OH}$. (b) Schematic drawing of the degradation behavior under in vitro assay for PVA/BaG/GA hybrids without crosslinking with surface erosion (SEM image). (c) Schematic drawing of the degradation behavior under in vitro assay for PVA/BaG/GA hybrids with 5.0\% GA crosslinking with bulk erosion (SEM image). (d) SEM image of the degradation behavior for PVA/BaG/GA hybrids with $1.0 \%$ GA crosslinking.

environment. There are numerous possibilities and pathways to be followed by each and every component. For instance, a component can undergo dissolution or solvation in the medium, erosion, etching, enzymatic, or hydrolytic cleavage of chains and networks into smaller species, among other possibilities. For this reason, it is beyond the focus of the present study to produce a complete in-depth investigation of the hybrids degradation process. On the contrary, it should be pointed out that a preliminary evaluation of the degradation behavior of hybrids (polymer-ceramic, PVA$\mathrm{BaG}$ ) was conducted in this research by performing an in vitro analysis based on mass loss upon immersion in aqueous medium. The main results are summarized in Figure 7. As shown in Figure 7(a), the mass loss values for all the samples proved to be higher at the beginning stages, followed by a progressive decrease in the mass loss rate according to immersion time. Moreover, the mass loss was higher for samples which were not chemically crosslinked with glutaraldehyde (PVA/BaG without GA, Figure $7(\mathrm{a})$, curve $(\mathrm{A})$ ). On the contrary, the mass loss was significantly reduced when the amount of chemical crosslinking agent was increased. For instance, after 21 days of in vitro assay, the mass loss varied from $\sim 50 \%$ for the noncrosslinked hybrid (Figure 7(a), curve (A)) to $~ 35 \%$ for hybrids crosslinked with $5.0 \%$ GA (Figure 7(a), curve (C)). In a general approach, two main regions can be clearly observed in Figure 7(a), one with a relatively high degradation rate (referred to as the $\mathrm{k} 1$ region) and the second with a lower degradation rate (k2). This finding may be associated with two concurrent mechanisms, one mostly dominated by solvation/dissolution (fast, $\mathrm{k} 1$ ) and the second by hydrolysis and diffusion (slow, k2) of the hybrid system. These results have given strong evidence that the original organic-inorganic network (PVA/BaG, Figure 7(c)) most likely was modified by the crosslinking reactions of PVA with the bifunctional reagent (GA), in turn causing the formation of acetal/hemiacetal bridges (Figure 7(b)) [34]. These results also suggested that the crosslinking of the hybrids improved the stability of scaffolds towards in vitro degradation. The PVA/BaG/GA scaffolds $(1.0 \%$ and $5.0 \%$ GA) degraded slowly, while PVA/BaG (0\% GA) degraded rapidly. Hence, these covalent bonds significantly altered the hybrid structure at the nanoscale level, thereby reducing the overall degradation rate. The schematic representation of the observed degradation behavior is illustrated in Figures 7(b) and $7(\mathrm{c})$, with the respective SEM images. The degradation led to some changes in the structural morphology as can be observed in the SEM micrographs (Figures 7(b) and 7(c)), which helps to explain the different degradation processes. Of course, this is a simplified approach, as many aspects are involved, such as network swelling, hydrogen bonds, hydrophobic and hydrophilic species, electrostatic balance of charges (ions), weak forces (van der Waals), among others. In fact, the choice of using water as the immersion medium rather than other degradation solutions, such as PBS (phosphate buffer saline) or SBF (simulated body fluid), was fundamentally justified by avoiding the effect of other ionic species in such already complex hybrid systems. 
This element warrants future investigation, since PBS is commonly used as a physiological medium for in vitro assays. A similar assay was carried out with hybrids crosslinked with $5.0 \%$ GA and previously submitted to a neutralizing treatment with an $\mathrm{NH}_{4} \mathrm{OH}$ solution before the degradation assay (Figure 7(a), curved). This assay, when compared to all samples, particularly with noncrosslinked samples, presented an even less pronounced mass loss ( $\sim 10 \%$ at 21 days) upon immersion. This result indicated that the treatment with an alkaline medium $\left(\mathrm{NH}_{4} \mathrm{OH}\right)$ not only neutralizes the hybrids, but also stabilizes the samples, perhaps removing part of the material susceptible to the initial stage of degradation [38]. Hence, when these samples were submitted to the alkaline neutralizing procedure, they underwent some preliminary erosion of the more labile components, achieving a more stable network.

Figure 8 presents a schematic illustration of the novel hybrid produced aiming at moving forward and bringing some contribution to this multifaceted subject of degradation of nanostructured materials. Briefly, hybrids are considered to be an interpenetrated network (IPN) produced by the combination of organic (PVA) and inorganic (BaG) constituents that have been chemically modified by a bifunctional crosslinker (GA). The degradation process can be described by a phenomenological contribution of solvation (dissolution) and diffusion-reaction mechanisms, occurring in two stages, as represented in Figure 8(b), Scheme 1, and Scheme 2 , respectively. Initially, an aqueous media diffuses into the hybrid structure which turns into a gradually swollen structure. In this stage (Figure 8(b), Scheme 1), it is expected that the effect of PVA as a water-soluble hydrophilic polymer will be mostly predominant (swelling/solvation polymeric component). It can also be said that temporary interactions (weak and strong) will be heavily affected at this stage, such as hydrogen bonds (hydroxyls of PVA and silanols), van der Waals forces (polymer chain folding), and electrostatic forces (ions, $\mathrm{Ca}^{2+}$, phosphates). In the sequence, within the hybrid polymeric-inorganic matrix, hydrolytic reactions may occur, mediated by water, forming some fragments and oligomeric products which may diffuse outwards. The diffusion depends on several factors, such as porosity, pore-size distribution and connectivity, and the degree of crosslinking of the nanostructured chemically modified hybrid network (Figure 8(b), Scheme 2). The complete erosion of the polymer is known to take substantially longer than the degradation time investigated in this study. As this hybrid network was modified by the chemical crosslinker, some comparable trends may be assumed at both degradation stages, but not necessarily to the same extent. During this first phase, the aqueous solution penetrates the $3 \mathrm{D}$ pore structure with several phenomena taking place, including swelling, solvation, and the leaching out of ions, among others. Then, at a second moment, some hydrolytic degradation may occur at the acetal and hemiacetal bridges, converting relatively long polymer chains (PVA) into shorter water-soluble fragments. Naturally, the degradation reaction of the chemically crosslinked organicinorganic systems will present a lower kinetic rate than will the dissolution of the noncrosslinked hybrid structure. In an analogous mechanism, the inorganic-rich component may also be degraded, which can be regarded as an inverse of the sol-gel poly-condensation process which occurred during the hybrid synthesis. In fact, several authors have reported on the degradation behavior of polymer-based systems, but very few detailed reports dealing specifically with the degradation of $3 \mathrm{D}$ porous hybrid scaffolds can be observed in the literature $[27,53]$.

In summary, based on the results, it is valid to affirm that the produced hybrid network of PVA-BaG was engineered according to the degradation kinetics by using different concentrations of bifunctional chemical covalent modifier. Furthermore, one may be able to tailor the rate of degradation by altering some accessible parameters, such as organic/inorganic ratio, scaffold porosity, and connectivity, type of crosslinker, among others, aimed at specific biomedical applications. Ideally, the degradation and resorption kinetics of composite and hybrid scaffolds are designed to allow cells to proliferate and secrete their own extracellular matrix while the scaffolds gradually vanish, leaving space for new cell and tissue growth.

\subsection{Cytotoxicity Assessment-In Vitro}

3.3.1. MTT Assay. Safety and reliable tests including cytotoxicity analyses are required for all products to be used in contact with humans and animals. Therefore, cell viability was measured using the MTT assay and represents the active mitochondrial enzymes present in a cell capable of reducing MTT. In general, MTT assays using cell culture have been accepted as the first step in identifying active compounds and for biosafety testing. As such, all synthesized hybrids of PVA/BaG were evaluated by MTT assay. Representative results are presented in Figure 9. A higher relative cytocompatibility of PVA/BaG hybrids crosslinked with 5.0\% GA, as compared to unmodified hybrids ( $0 \% \mathrm{GA})$, can be observed. Moreover, samples submitted to the in vitro degradation process have clearly indicated an improvement in their preliminary biocompatibility during longer immersion times (21 days). Taking into consideration all the results, the PVA/BaG/GA hybrid crosslinked with 5.0\% GA presented the best performance in the MTT assay. Similar results were verified for the PVA/BaG/GA hybrid crosslinked with 5.0\% GA, which had been previously neutralized by $\mathrm{NH}_{4} \mathrm{OH}$ medium. Interestingly, these very hybrids have also shown better results in terms of structure and mechanical behavior. They presented a more homogeneous pore size distribution; a higher yield strength and strain deformation; and a lower degradation rate. Nevertheless, one should be aware that the values found in MTT results are attributed to a comparable biocompatibility under in vitro conditions and cannot be straightforwardly used to predict in vivo performance in biomedical applications.

3.3.2. Cell Viability via Adhesion-Spreading Assay. Moving beyond the MTT assay, the qualitative analysis of cell morphology was conducted by means of SEM images (Figure 9(b)) associated with its proliferation and growth in 


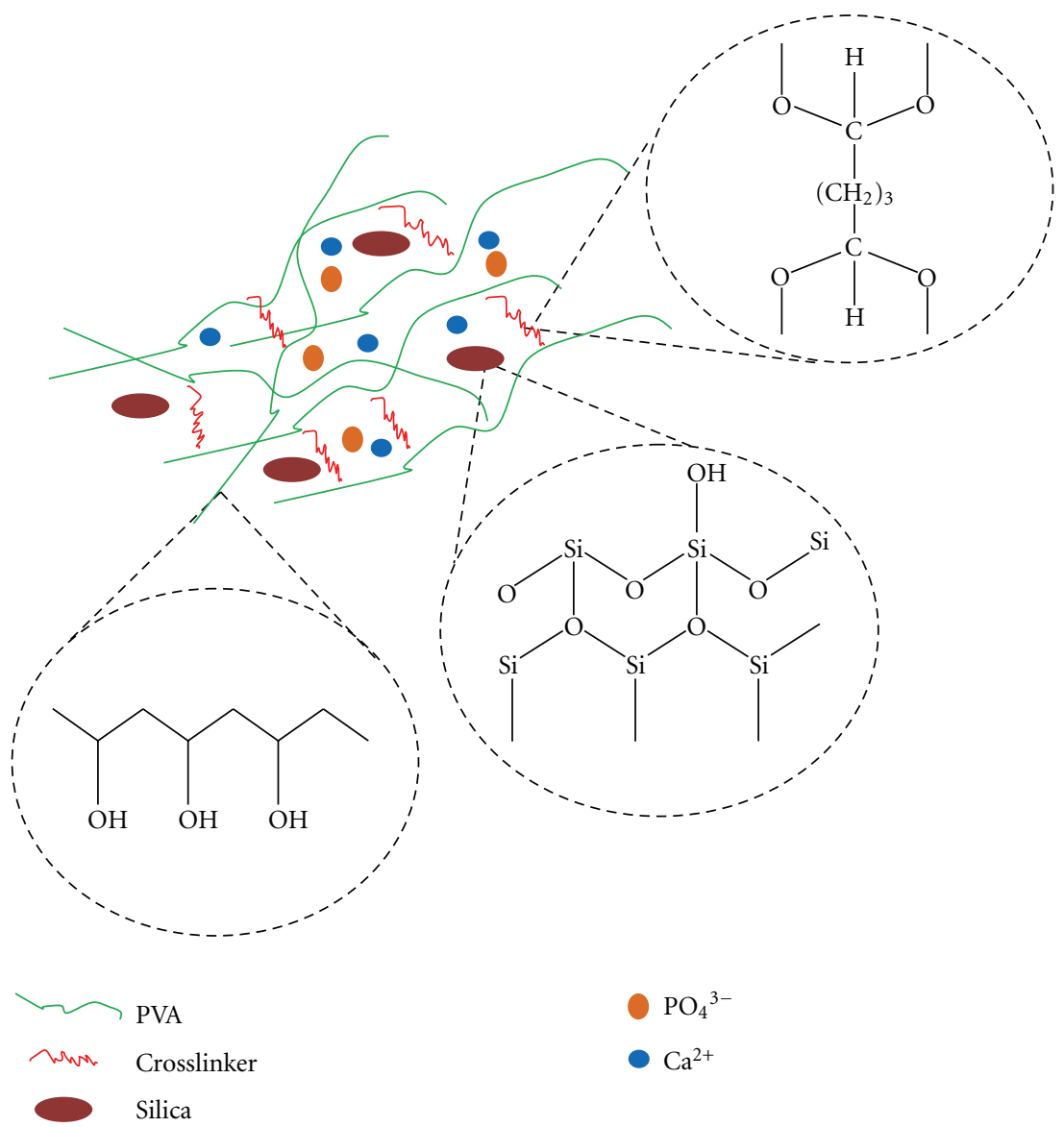

(a)

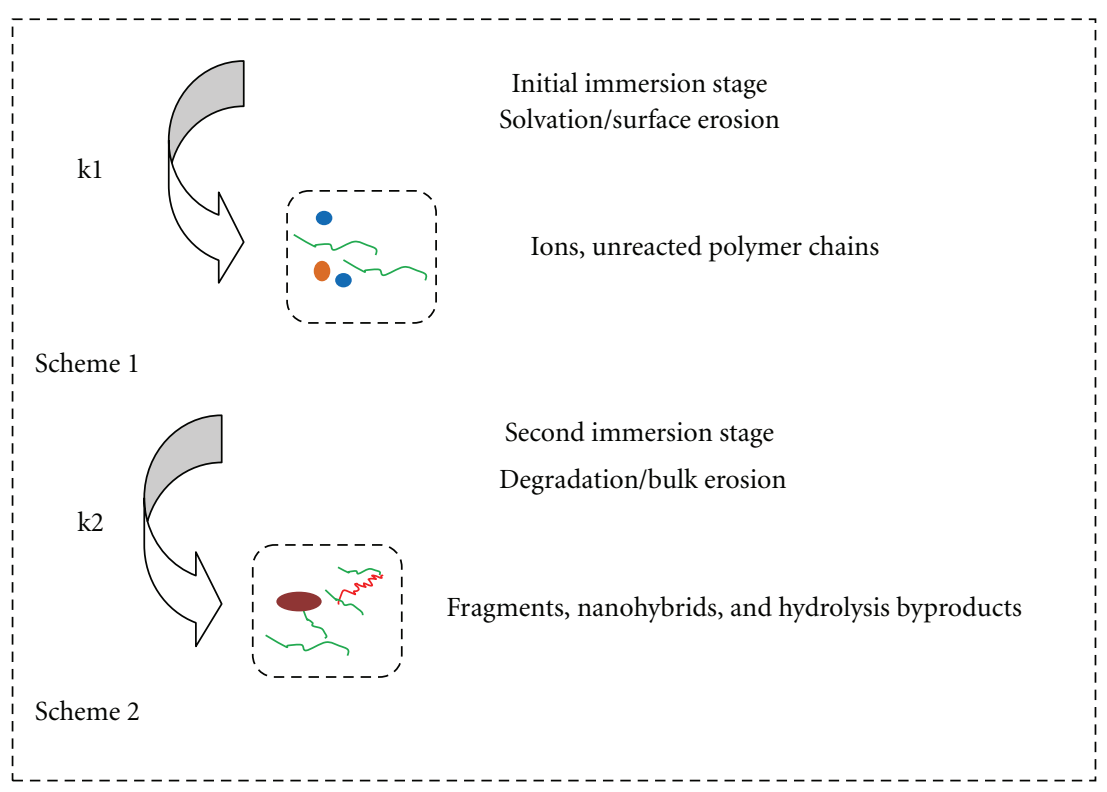

(b)

Figure 8: (a) Representation of the complex nanostructure of the hybrids based on PVA/BaG/GA. Surface erosion of noncrosslinked hybrids associated with solvation/dissolution at fast rate k1 (Scheme 1); Bulk erosion of hybrids chemically modified with bifunctional crosslinker at slow rate $\mathrm{k} 2$ (Scheme 2). 


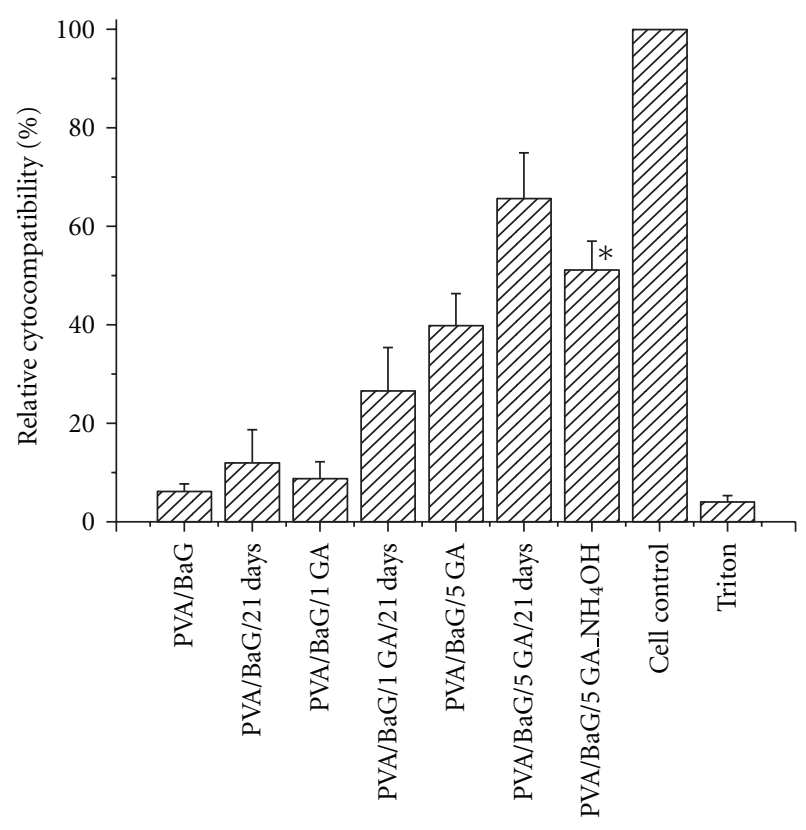

(a)

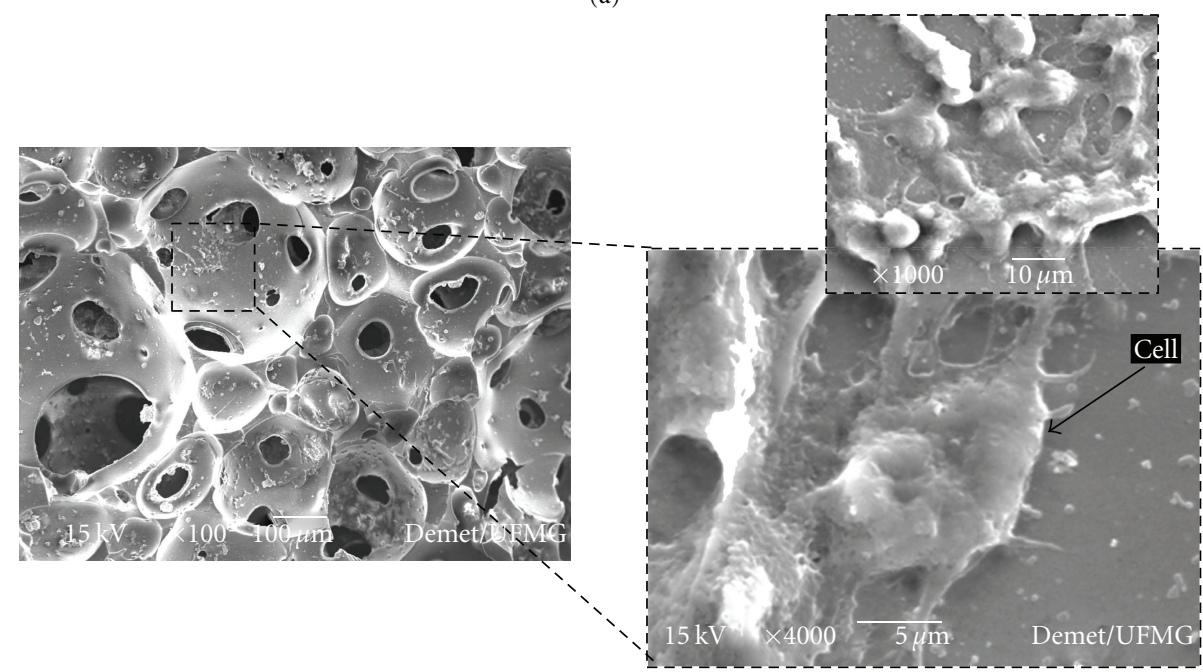

(b)

(c)

FIGURE 9: (a) Typical cell viability response of MTT assay of PVA/BaG and PVA/BaG/GA hybrids crosslinked with $1.0 \%$ and 5.0\% GA before and after in vitro degradation (21 days). Also, PVA/BaG/GA (5.0\%) with $\mathrm{NH}_{4} \mathrm{OH}$ stabilization process. SEM images of hybrid scaffolds crosslinked at different magnifications with evidence of cell adhesion/spreading (b, c) (* see [38]).

contact with PVA/BaG/GA hybrid crosslinked with 5.0\% GA samples via adhesion and spreading behavior.

It could be verified that the VERO cells seeded onto PVA/BaG/GA hybrid matrices showed a good adhesion and spreading morphology, that is, well matched to this fibroblastic lineage (Figure 9(b)). Since cellular attachment, adhesion, and spreading belong to the first phase of cell/material interactions, the quality of this phase will influence the proliferation and differentiation of cells on biomaterials surfaces. Based on the SEM results, one may attribute the VERO cell spreading and adhesion verified in the PVA/BaG/GA hybrid to be an important response regarding the biocompatibility and noncytotoxicity of the investigated samples. According to the literature $[30,36,38]$, cell spreading is generally divided into three main interaction levels: (a) unspread: cells were still spherical in appearance, and protrusions or lamellipodia were not yet produced; (b) partially spread: at this stage, cells began to spread laterally on one or more sides, but the extensions of the plasma membrane were not completely confluent; (c) fully spread. The final model (c) would represent the best result for material cell hosting. These morphological aspects are accredited to healthy VERO cell cultures giving clear evidence of hybrids produced cytocompatibility. Additionally, the selected cell line (VERO) used as a model in this research plays a role in producing many of the components that are essential to the connective tissue, for example, extracellular components, such as glycosaminoglycans, and fibrous tissue, such as 
collagen. In summary, cell adhesion and spreading are of vital importance in living biological processes and are involved in various natural phenomena, such as embryogenesis, the maintenance of tissue structure, wound healing, immune response, metastasis, and tissue integration of biomaterials $[30,36,38,42,43]$.

\section{Conclusions}

Three-dimensional interconnected porous structures comprised of PVA/BaG were obtained with varying structural and mechanical properties for different degrees of network crosslinking. A decrease in the medium pore and interconnection size and a more homogeneous pore size distribution, with $80 \%$ within the range of $100-450 \mu \mathrm{m}$, was observed for larger concentrations of glutaraldehyde. In addition, an increase in the yield strength and in the deformation at the yield stress, with an increase in crosslinking, could be observed, which proved to be more pronounced for the sample crosslinked with 5.0\% GA. The synthesized organic-inorganic hybrids based on PVA/BaG have presented different degradation kinetics due to the degree of chemical crosslinking which altered the nanostructure of the network. Finally, these engineered hybrids have shown a preliminary cell viability behavior that, when combined with appropriate mechanical and morphological properties, can be considered potential biomaterials for tissue engineering applications.

\section{Acknowledgments}

They acknowledge the financial support from CAPES, FAPEMIG, and CNPq. The authors would like to express their special gratitude to Professor Edel F. Barbosa-Stancioli (ICB-UFMG Brazil) for the MTT cytotoxicity assays and Dr. Julian R. Jones (Imperial College London UK) for the $\mu \mathrm{CT}$ analysis.

\section{References}

[1] J. D. Kretlow and A. G. Mikos, "From material to tissue: biomaterial development, scaffold fabrication, and tissue engineering," AIChE Journal, vol. 54, no. 12, pp. 3048-3067, 2008.

[2] U. A. Stock and J. P. Vacanti, "Tissue engineering: current state and prospects," Annual Review of Medicine, vol. 52, pp. 443451, 2001.

[3] J. J. Blaker, S. N. Nazhat, V. Maquet, and A. R. Boccaccini, "Long-term in vitro degradation of PDLLA/Bioglass $(\mathrm{B}$ bone scaffolds in acellular simulated body fluid," Acta Biomaterialia, vol. 7, no. 2, pp. 829-840, 2011.

[4] Y. Chen, S. Zhou, and Q. Li, "Mathematical modeling of degradation for bulk-erosive polymers: applications in tissue engineering scaffolds and drug delivery systems," Acta Biomaterialia, vol. 7, no. 3, pp. 1140-1149, 2011.

[5] P. Fabbri, V. Cannillo, A. Sola, A. Dorigato, and F. Chiellini, "Highly porous polycaprolactone-45S5 Bioglass $\AA$ scaffolds for bone tissue engineering," Composites Science and Technology, vol. 70, no. 13, pp. 1869-1878, 2010.
[6] Y. Minaberry and M. Jobbágy, "Macroporous bioglass scaffolds prepared by coupling sol-gel with freeze drying," Chemistry of Materials, vol. 23, no. 9, pp. 2327-2332, 2011.

[7] L.-C. Gerhardt and A. R. Boccaccini, "Bioactive glass and glass-ceramic scaffolds for bone tissue engineering," Materials, vol. 3, pp. 3867-3910, 2010.

[8] J. J. Blaker, A. Bismarck, A. R. Boccaccini, A. M. Young, and S. N. Nazhat, "Premature degradation of $\operatorname{poly}(\alpha$-hydroxyesters) during thermal processing of Bioglass $\mathrm{B}$-containing composites," Acta Biomaterialia, vol. 6, no. 3, pp. 756-762, 2010.

[9] Q. Tan, S. Li, J. Ren, and C. Chen, "Fabrication of porous scaffolds with a controllable microstructure and mechanical properties by porogen fusion technique," International Journal of Molecular Sciences, vol. 12, no. 2, pp. 890-904, 2011.

[10] E. M. Valliant and J. R. Jones, "Softening bioactive glass for bone regeneration: sol-gel hybrid materials," Soft Matter, vol. 7, no. 11, pp. 5083-5095, 2011.

[11] Q. Fu, M. N. Rahaman, H. Fu, and X. Liu, "Silicate, borosilicate, and borate bioactive glass scaffolds with controllable degradation rate for bone tissue engineering applications. I. Preparation and in vitro degradation," Journal of Biomedical Materials Research-Part A, vol. 95, no. 1, pp. 164-171, 2010.

[12] K. J. L. Burg, S. Porter, and J. F. Kellam, "Biomaterial developments for bone tissue engineering," Biomaterials, vol. 21, no. 23, pp. 2347-2359, 2000.

[13] D. W. Hutmacher, J. T. Schantz, C. X. Lam, K. C. Tan, and T. C. Lim, "State of the art and future directions of scaffold-based bone engineering from a biomaterials perspective," Journal of Tissue Engineering and Regenerative Medicine, vol. 1, no. 4, pp. 245-260, 2007.

[14] V. Olivier, N. Faucheux, and P. Hardouin, "Biomaterial challenges and approaches to stem cell use in bone reconstructive surgery," Drug Discovery Today, vol. 9, no. 18, pp. 803-811, 2004.

[15] J. R. Jones and L. L. Hench, "Regeneration of trabecular bone using porous ceramics," Current Opinion in Solid State and Materials Science, vol. 7, no. 4-5, pp. 301-307, 2003.

[16] M. Vallet-Regí and J. M. González-Calbet, "Calcium phosphates as substitution of bone tissues," Progress in Solid State Chemistry, vol. 32, no. 1-2, pp. 1-31, 2004.

[17] W. Paul and P. Sharma, "Nanoceramic matrices: biomedical applications," American Journal of Biochemistry and Biotechnology, vol. 2, pp. 41-48, 2006.

[18] J. R. Jones, L. M. Ehrenfried, and L. L. Hench, "Optimising bioactive glass scaffolds for bone tissue engineering," Biomaterials, vol. 27, no. 7, pp. 964-973, 2006.

[19] S. Padilla, S. Sánchez-Salcedo, and M. Vallet-Regí, "Bioactive glass as precursor of designed-architecture scaffolds for tissue engineering," Journal of Biomedical Materials Research-Part A, vol. 81, no. 1, pp. 224-232, 2007.

[20] X. Yan, C. Yu, X. Zhou, J. Tang, and D. Zhao, "Highly ordered mesoporous bioactive glasses with superior in vitro boneforming bioactivities," Angewandte Chemie-International Edition, vol. 43, no. 44, pp. 5980-5984, 2004.

[21] J. E. Gough, J. R. Jones, and L. L. Hench, "Nodule formation and mineralisation of human primary osteoblasts cultured on a porous bioactive glass scaffold," Biomaterials, vol. 25, no. 11, pp. 2039-2046, 2004.

[22] P. Valerio, M. M. Pereira, A. M. Goes, and M. F. Leite, "The effect of ionic products from bioactive glass dissolution on osteoblast proliferation and collagen production," Biomaterials, vol. 25, no. 15, pp. 2941-2948, 2004.

[23] I. D. Xynos, A. J. Edgar, L. D. K. Buttery, L. L. Hench, and J. M. Polak, "Ionic products of bioactive glass dissolution increase 
proliferation of human osteoblasts and induce insulin-like growth factor II mRNA expression and protein synthesis," Biochemical and Biophysical Research Communications, vol. 276, no. 2, pp. 461-465, 2000.

[24] H. Mansur, R. Oréfice, M. Pereira, Z. Lobato, W. Vasconcelos, and L. Machado, "FTIR and UV-vis study of chemically engineered biomaterial surfaces for protein immobilization," Spectroscopy, vol. 16, no. 3-4, pp. 351-360, 2002.

[25] G. Andrade, E. F. Barbosa-Stancioli, A. A. Piscitelli Mansur, W. L. Vasconcelos, and H. S. Mansur, "Design of novel hybrid organic-inorganic nanostructured biomaterials for immunoassay applications," Biomedical Materials, vol. 1, no. 4, article 008, pp. 221-234, 2006.

[26] V. M. Bispo, A. A. P. Mansur, E. F. Barbosa-Stancioli, and H. S. Mansur, "Biocompatibility of nanostructured chitosan/poly(vinyl alcohol) blends chemically crosslinked with genipin for biomedical applications," Journal of Biomedical Nanotechnology, vol. 6, no. 2, pp. 166-175, 2010.

[27] K. Rezwan, Q. Z. Chen, J. J. Blaker, and A. R. Boccaccini, "Biodegradable and bioactive porous polymer/inorganic composite scaffolds for bone tissue engineering," Biomaterials, vol. 27, no. 18, pp. 3413-3431, 2006.

[28] S. Verrier, J. J. Blaker, V. Maquet, L. L. Hench, and A. R. Boccaccini, "PDLLA/Bioglass $(\mathrm{B}$ composites for soft-tissue and hard-tissue engineering: an in vitro cell biology assessment," Biomaterials, vol. 25, no. 15, pp. 3013-3021, 2004.

[29] A. Stein, B. J. Melde, and R. C. Schroden, "Hybrid inorganicorganic mesoporous silicates-nanoscopic reactors coming of age," Advanced Materials, vol. 12, no. 19, pp. 1403-1419, 2000.

[30] H. S. Mansur and H. S. Costa, "Nanostructured poly(vinyl alcohol)/bioactive glass and poly(vinyl alcohol)/chitosan/ bioactive glass hybrid scaffolds for biomedical applications," Chemical Engineering Journal, vol. 137, no. 1, pp. 72-83, 2008.

[31] J. R. Jones, "New trends in bioactive scaffolds: the importance of nanostructure," Journal of the European Ceramic Society, vol. 29, no. 7, pp. 1275-1281, 2009.

[32] T. Yamaoka, Y. Tabata, and Y. Ikada, "Comparison of body distribution of poly(vinyl alcohol) with other water-soluble polymers after intravenous administration," Journal of Pharmacy and Pharmacology, vol. 47, no. 6, pp. 479-486, 1995.

[33] E. Chiellini, A. Corti, S. D’Antone, and R. Solaro, "Biodegradation of poly (vinyl alcohol) based materials," Progress in Polymer Science, vol. 28, no. 6, pp. 963-1014, 2003.

[34] H. S. Mansur, C. M. Sadahira, A. N. Souza, and A. A. P. Mansur, "FTIR spectroscopy characterization of poly (vinyl alcohol) hydrogel with different hydrolysis degree and chemically crosslinked with glutaraldehyde," Materials Science and Engineering C, vol. 28, no. 4, pp. 539-548, 2008.

[35] M. M. Pereira, J. R. Jones, and L. L. Hench, "Bioactive glass and hybrid scaffolds prepared by sol-gel method for bone tissue engineering," Advances in Applied Ceramics, vol. 104, no. 1, pp. 35-42, 2005.

[36] H. S. Costa, M. F. Rocha, G. I. Andrade et al., "Sol-gel derived composite from bioactive glass-polyvinyl alcohol," Journal of Materials Science, vol. 43, no. 2, pp. 494-502, 2008.

[37] A. A. R. De Oliveira, V. Ciminelli, M. S. S. Dantas, H. S. Mansur, and M. M. Pereira, "Acid character control of bioactive glass/polyvinyl alcohol hybrid foams produced by sol-gel," Journal of Sol-Gel Science and Technology, vol. 47, no. 3, pp. 335-346, 2008.

[38] H. S. Costa, E. F. B. Stancioli, M. M. Pereira, R. L. Oréfice, and H. S. Mansur, "Synthesis, neutralization and blocking procedures of organic/inorganic hybrid scaffolds for bone tissue engineering applications," Journal of Materials Science, vol. 20, no. 2, pp. 529-535, 2009.

[39] E. S. Costa-Júnior, E. F. Barbosa-Stancioli, A. A. P. Mansur, W. L. Vasconcelos, and H. S. Mansur, "Preparation and characterization of chitosan/poly(vinyl alcohol) chemically crosslinked blends for biomedical applications," Carbohydrate Polymers, vol. 76, no. 3, pp. 472-481, 2009.

[40] J. A. Burdick, C. Chung, X. Jia, M. A. Randolph, and R. Langer, "Controlled degradation and mechanical behavior of photopolymerized hyaluronic acid networks," Biomacromolecules, vol. 6, no. 1, pp. 386-391, 2005.

[41] H. S. Mansur, R. L. Oréfice, and A. A. P. Mansur, "Characterization of poly(vinyl alcohol)/poly(ethylene glycol) hydrogels and PVA-derived hybrids by small-angle X-ray scattering and FTIR spectroscopy," Polymer, vol. 45, no. 21, pp. 7193-7202, 2004.

[42] P. Estacia, A. S. Rodrigues Junior, P. L. Moreira, and S. C. Genari, "The cytotoxicity in vero cells of a perfluorocarbon used in vitreoretinal surgery," Brazilian Journal of Morphological Sciences, vol. 19, no. 2, pp. 41-47, 2002.

[43] T. Mosmann, "Rapid colorimetric assay for cellular growth and survival: application to proliferation and cytotoxicity assays," Journal of Immunological Methods, vol. 65, no. 1-2, pp. 55-63, 1983.

[44] J. Coates, "Encyclopedia of analytical chemistry: interpretation of infrared spectra, a practical approach," in Encyclopedia of Analytical Chemistry, R. A. Meyers, Ed., pp. 10815-10837, John Wiley \& Sons, Chichester, UK, 2000.

[45] R. M. Almeida and C. G. Pantano, "Structural investigation of silica gel films by infrared spectroscopy," Journal of Applied Physics, vol. 68, no. 8, article 4225, 8 pages, 1990.

[46] J. Román, S. Padilla, and M. Vallet-Regí, "Sol-gel glasses as precursors of bioactive glass ceramics," Chemistry of Materials, vol. 15, no. 3, pp. 798-806, 2003.

[47] A. I. Martín, A. J. Salinas, and M. Vallet-Regí, "Bioactive and degradable organic-inorganic hybrids," Journal of the European Ceramic Society, vol. 25, no. 16, pp. 3533-3538, 2005.

[48] T. Wang, M. Turhan, and S. Gunasekaran, "Selected properties of pH-sensitive, biodegradable chitosan-poly(vinyl alcohol) hydrogel," Polymer International, vol. 53, no. 7, pp. 911-918, 2004.

[49] S.-H. Shin and H.-I. Kim, "Contribution of hydrogen bond and coupling reaction improvement in compatibility of organic polymer/silica nanocomposites," Industrial \& Engineering Chemistry Research, vol. 7, pp. 147-152, 2001.

[50] S. T. Ho and D. W. Hutmacher, "A comparison of micro CT with other techniques used in the characterization of scaffolds," Biomaterials, vol. 27, no. 8, pp. 1362-1376, 2006.

[51] L. J. Gibson, "Biomechanics of cellular solids," Journal of Biomechanics, vol. 38, no. 3, pp. 377-399, 2005.

[52] H. Costa, A. A. R. De Oliveira, R. L. Oréfice, H. S. Mansur, and M. M. Pereira, "Tailoring mechanical behavior of PVAbioactive glass hybrid foams," Key Engineering Materials, vol. 361-363, pp. 289-292, 2008.

[53] M. Vert, S. M. Li, G. Spenlehauer, and P. Guerin, "Bioresorbability and biocompatibility of aliphatic polyesters," Journal of Materials Science, vol. 3, no. 6, pp. 432-446, 1992. 

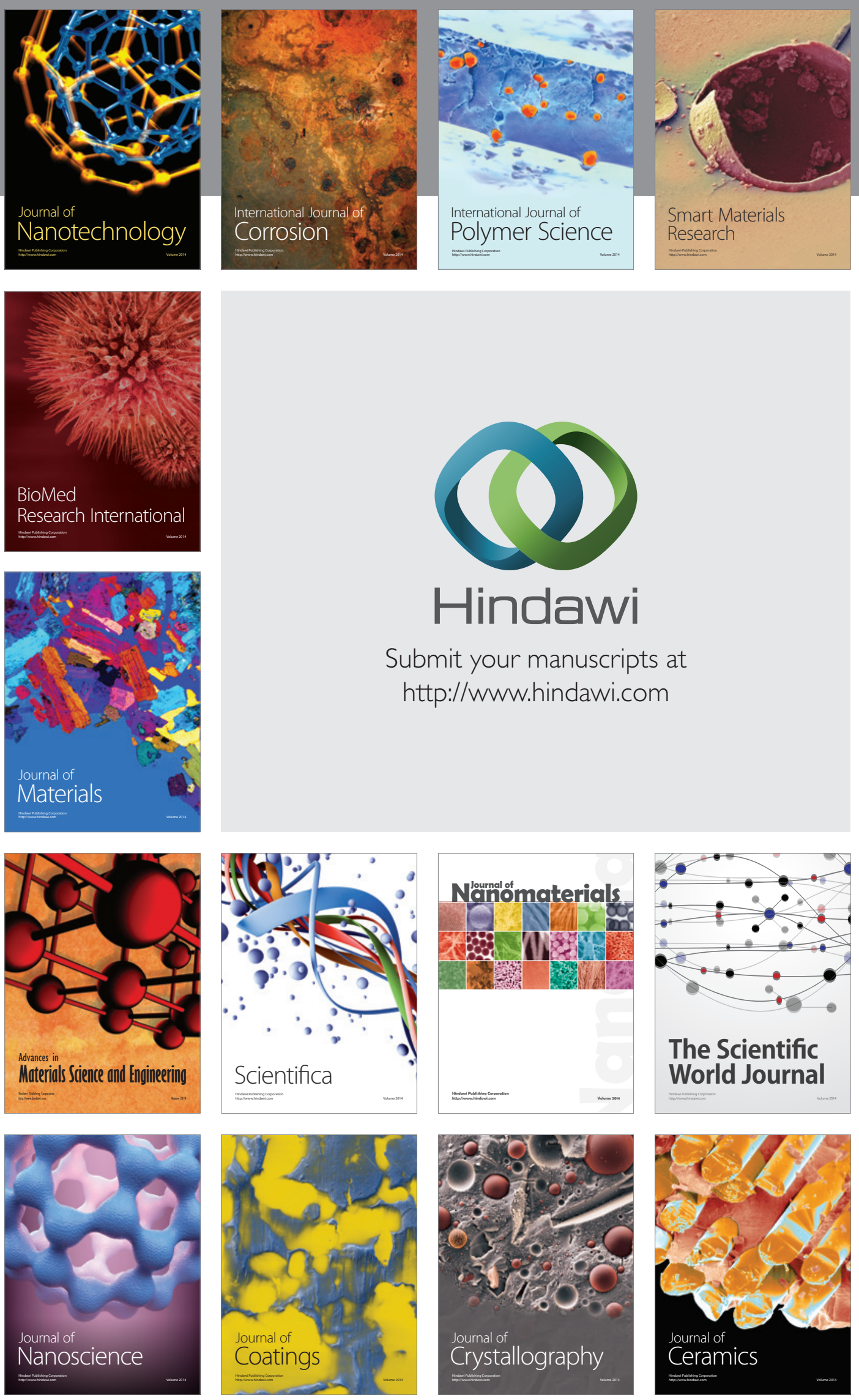

The Scientific World Journal

Submit your manuscripts at

http://www.hindawi.com

\section{World Journal}

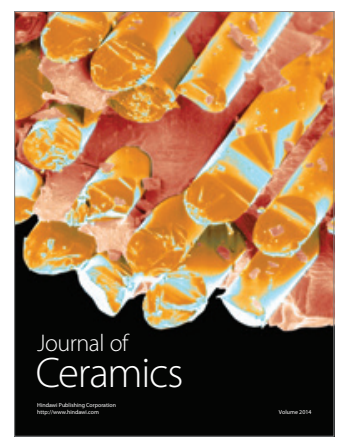

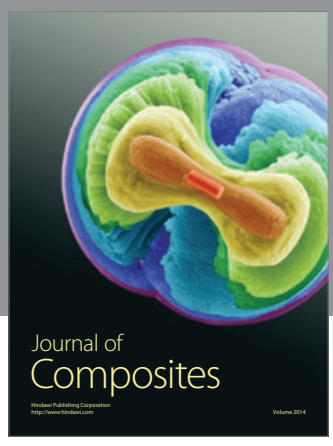
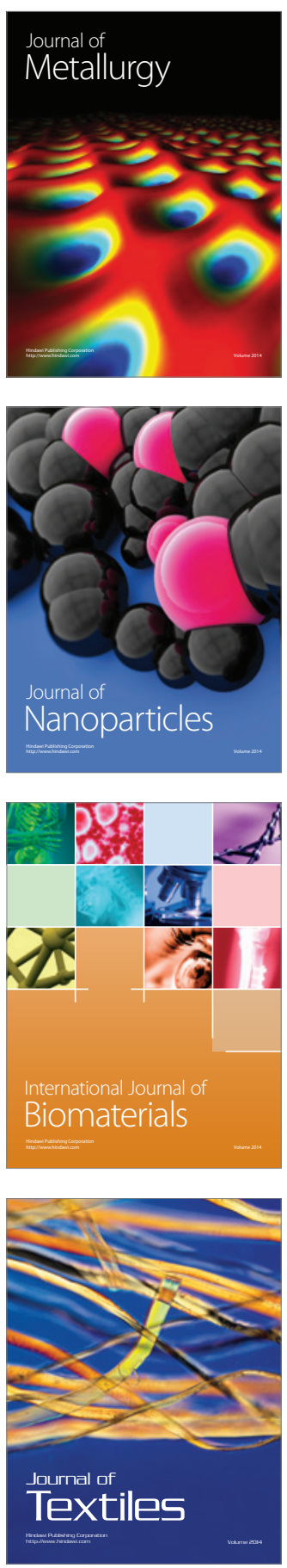